\title{
Novel Speed-and-Power-Optimized SRAM Cell Designs with Enhanced Self-Recoverability from Single- and Double-Node Upsets
}

\author{
Aibin Yan, Yan Chen, Yuanjie Hu, Jun Zhou, Tianming Ni, Jie Cui, Patrick Girard, Fellow, IEEE, \\ and Xiaoqing Wen, Fellow, IEEE
}

\begin{abstract}
The continuous advancement of CMOS technologies makes SRAMs more and more sensitive to soft errors. This paper presents two novel radiation-hardened SRAM cell designs, namely S4P8N and S8P4N, with enhanced self-recoverability from single-node upsets (SNUs) and Double-node upsets (DNUs). First, the S4P8N cell that has more redundant nodes and more access transistors is proposed. The cell has the following advantages: (1) it can self-recover from all possible SNUs; (2) it can self-recover from a part of DNUs; (3) it has small overhead in terms of power dissipation. Then, to reduce read and write access time, the $S 8 P 4 N$ cell that uses a special feedback mechanism among its internal nodes is proposed. The cell has similar soft error tolerability as the S4P8N cell. Simulation results validate the high robustness of the proposed SRAM cells. These results also show that the write access time, read access time, and power dissipation of the $S 8 \mathrm{P} 4 \mathrm{~N}$ cell are reduced approximately by $29 \%$, $20 \%$, and $21 \%$ on average, at the cost of moderate silicon area, when compared with the state-of-the-art radiation-hardened SRAM cells.
\end{abstract}

Index Terms-SRAM; radiation hardening; soft error; self-recoverability; node upset

\section{INTRODUCTION}

A S CMOS technology scaling, SRAMs can achieve high density, low power consumption and high performance. However, this pushes integrated circuit (IC) designers and technologists to increasingly focus on SRAM reliability, since small transistor feature sizes also give rise to low supply voltages and small node capacitances that can increase the sensitivity of SRAMs to particle-striking-induced soft errors

Manuscript received February 6, 2020; revised ???; accepted ??? . Date of publication ???; This work was supported in part by the National Natural Science Foundation of China (61974001, 61874156, 61674048, 61904001, and 61834006).

Aibin Yan, Yan Chen, Yuanjie Hu, Jun Zhou, and Jie Cui are with the Key Laboratory of Intelligent Computing and Signal Processing of Ministry of Education, and also with the School of Computer Science and Technology, Anhui University, Hefei 230601, China (E-mail: \{abyan, cuijie\}@mail.ustc.edu.cn, \{yanchenvv, huyj0115, zhoujun_3199\}@qq.com).

Tianming $\mathrm{Ni}$ is with the College of Electrical Engineering, Anhui Polytechnic University, Wuhu 241000, China. (E-mail: timmyni126@126.com). He is the corresponding author.

Patrick Girard is with the Laboratory of Informatics, Robotics and Microelectronics of Montpellier, University of Montpellier / CNRS, Montpellier 34095, France (E-mail: girard@lirmm.fr).

Xiaoqing Wen is with the Graduate School of Computer Science and Systems Engineering, Kyushu Institute of Technology, Fukuoka 820-8502, Japan (E-mail: wen@cse.kyutech.ac.jp)
[1]. It is reported in [2] that high-energy particles, such as protons, neutrons, heavy ions, electrons, muons and alpha particles, including kinds of rays, can severely impact the reliability of advanced nano-scale SRAMs. That is, soft errors can invalidly change the values stored in SRAMs. Although the recently adopted FinFET technology can reduce the soft error rate at transistor or cell level [3], effective and scalable solutions for soft error tolerance are still needed. Therefore, there is a strong need for IC designers to mitigate soft-error-induced reliability issues through designing novel radiation-hardened structures.

When a particle strikes an OFF-state transistor in a logic gate, a dense track of electron-hole pairs can be generated. As a result, a transient pulse, i.e., a single event transient (SET) pulse, can be produced at the node that collects the charge and the transient pulse can be detected at the output of the affected logic gate. If the SET pulse propagates through downstream combinational logic gates and arrives at a storage element, the pulse may be captured and causes invalid value-retention in the storage element [4]. In the same context, a particle may directly strike an OFF-state transistor in a storage element, causing a node-value change, which is called a single node upset $(S N U)$. Moreover, transistor feature sizes are becoming smaller and smaller, leading to reduced spacing between nodes. Hence, in a circuit, both OFF-state transistors may be affected by one striking-particle simultaneously because of multiple-node charge-collection mechanisms [5], and thus a double-node upset $(D N U)$ may occur. In summary, SRAMs may store incorrect values because of SNUs and/or DNUs especially for advanced nano-scale CMOS technologies, resulting in an increasing challenge for IC designers to mitigate reliability issues.

To mitigate SNUs and/or DNUs, IC designers have proposed several novel designs of latches [6-8], flip-flops [9-11] and SRAMs [3, 12-28] through the radiation hardening by design (RHBD) approach. This paper mainly considers hardening for SRAMs. The conventional SRAM, namely $6 \mathrm{~T}$, consists of six transistors, i.e., it includes two PMOS transistors and two NMOS transistors to store values and two NMOS transistors for access operations. Since the 6T SRAM cannot tolerate SNUs, many hardened SRAMs have been proposed to tolerate SNUs. Typical SNU-hardened SRAMs include NASA13T [13], QUCCE10T [14], QUCCE12T [14], We-Quatro [15], PS10T [16], Lin12T [17], and RH12T [18]. 
However, these SRAMs still have the following disadvantages.

(1) Some SRAMs cannot provide complete SNU self-recoverability [13-15].

(2) Some SRAMs have large overhead especially in terms of read access time [14, 16-18], write access time [14, 16] and power dissipation $[13,14]$.

(3) To mitigate SNUs, some SRAMs have to use extra techniques such as sizing up some transistors [13], identifying sensitive and insensitive nodes [14]. These solutions increase the area overhead and design complexity.

Our previous work in [19] has very large overhead; our previous work in [12] has at least one node that cannot self-recover from an SNU; these issues motivate us to propose SRAM cells that have low overhead but advanced self-recoverability. Based on the RHBD approach, this paper first presents a novel reliable SRAM cell whose storage part includes 4 PMOS and 8 NMOS transistors (namely S4P8N cell) hardened against SNUs and DNUs. Due to the elaborately constructed error-interceptive feedback loops, the cell can self-recover from all possible SNUs and a part of DNUs regardless of the energy of striking-particles. To reduce read and write access time, an access-operation optimized SRAM cell whose storage part includes 8 PMOS and 4 NMOS transistors (namely S8P4N cell) is further proposed. The cell has a similar soft error tolerance capability as the S4P8N cell. Moreover, owing to the use of special feedback mechanisms among internal nodes, the proposed cells have low power dissipation. Indeed, each of the cells has two interlocked feedback loops. So, there is no current competition inside (and between) the feedback loops. Moreover, the feedback loops are complementary since, as soon as one feedback loop is activated, the other feedback loop will be also activated. Simulation results demonstrate the reliability and cost-effectiveness of the proposed SRAM cells.

The rest of this paper is organized as follows. Section II presents the schematic and working principles of the proposed S4P8N cell. Section III presents the schematic and working principles of the proposed S8P4N cell. Section IV provides comparison and evaluation results about reliability and overhead for SRAM cells, and Section V concludes the paper.

It should be noted that, for fault injection simulations in the subsequent sections, a flexible dual-exponential current-source model was used [29]. In the simulations, the time constant of the rise and fall of the current pulse was set to 0.1 and $3.0 \mathrm{ps,}$ respectively. The worst case injected charge was chosen to be up to $25 \mathrm{fC}$ for a single node, which was large enough. All the simulations of the proposed S4P8N and S8P4N cells were performed using the Synopsys HSPICE tool, with an advanced 22nm CMOS library from GlobalFoundries under room temperature, and the supply voltage was set to $0.8 \mathrm{~V}$.

\section{PROPOSED S4P8N SRAM CELL}

Figure 1 shows a conceptual view of the proposed SRAMs to enhance self-recoverability from SNUs and DNUs and improve read-access and write-access times. We use two interlocked one-PMOS-transistor-and-one-NMOS-transistor
$(1 \mathrm{P} 1 \mathrm{~N})$ storage blocks (feedback loops) to store values and use

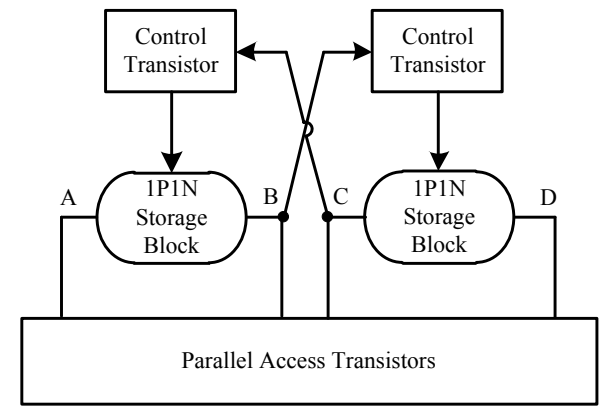

Fig. 1. Conceptual view of the proposed SRAM cells.

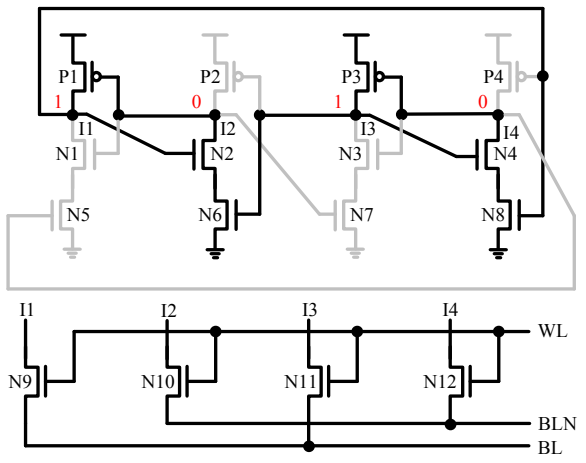

Fig. 2. Schematic of the proposed S4P8N cell.

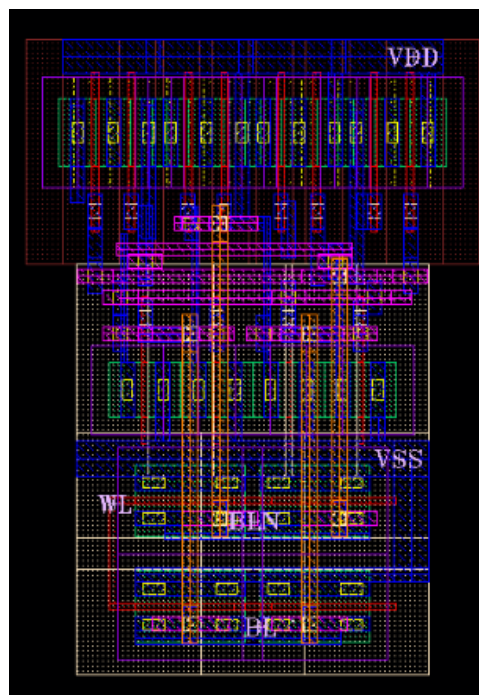

Fig. 3. Layout of the proposed S4P8N cell.

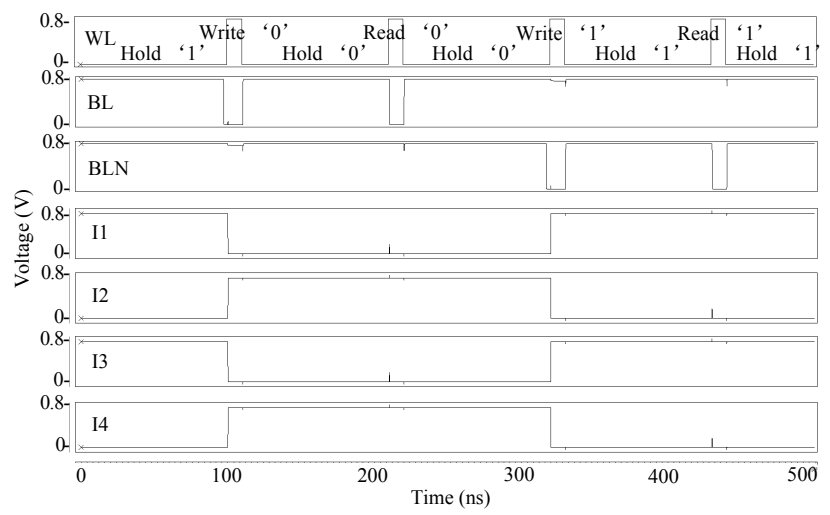

Fig. 4. Simulation results for normal operations of the proposed S4P8N cell. 
parallel access transistors to improve access operation. To enhance self-recoverability from SNUs and DNUs, one of the blocks is controlled by the other through a control transistor. The fact that there is no current competition inside (and between) the blocks can also improve access operation. The schematic of the proposed S4P8N cell is depicted in Fig. 2. The S4P8N cell consists of 16 transistors, i.e., PMOS transistors $\mathrm{P} 1$ to $\mathrm{P} 4$ and NMOS transistors $\mathrm{N} 1$ to N12. The storage part of the cell has 4 PMOS transistors and 8 NMOS transistors, i.e., transistors $\mathrm{P} 1$ to $\mathrm{P} 4$ and $\mathrm{N} 1$ to N8. Transistors N9 to N12 are access transistors that are controlled by word line WL. In the proposed S4P8N cell, I1 to I4 are internal nodes and they are connected to bit lines BL and BLN through access transistors N9 to N12, respectively. Figure 3 shows the layout of the proposed $\mathrm{S} 4 \mathrm{P} 8 \mathrm{~N}$ cell. When $\mathrm{WL}=1$, all access transistors are $\mathrm{ON}$, and write/read access operations can be executed. When $\mathrm{WL}=0$, the access transistors are OFF, and the cell retains the stored value.

The normal operations of the proposed S4P8N cell are described as follows. Figure 2 shows the scenario when the $\mathrm{S} 4 \mathrm{P} 8 \mathrm{~N}$ cell stores 1 , i.e., $\mathrm{I} 1=\mathrm{I} 3=1$ and $\mathrm{I} 2=\mathrm{I} 4=0$.

(1) We consider the case of writing 1 . At this time, BL is high while $\mathrm{BLN}$ is low. When WL is changed to 1 , transistors P1, P3, N2, N4, N6, and N8 become ON and transistors P2, $\mathrm{P} 4, \mathrm{~N} 1, \mathrm{~N} 3, \mathrm{~N} 5$, and N7 become OFF, and a 1 is written into the S4P8N cell. Since feedback loops are constructed in the S4P8N cell, the cell can retain the value through the feedback loops (i.e., I1 $\rightarrow$ I4 $\rightarrow$ I3 $\rightarrow$ I2 $\rightarrow$ I1, I2 $\rightarrow$ I1 $\rightarrow$ I2, and I3 $\rightarrow$ I4 $\rightarrow$ I3).

(2) The hold operation is considered when storing 1 . At this time, $\mathrm{WL}=0$, transistors $\mathrm{P} 1, \mathrm{P} 3, \mathrm{~N} 2, \mathrm{~N} 4, \mathrm{~N} 6$, and $\mathrm{N} 8$ are $\mathrm{ON}$ while the other transistors are OFF. Hence, the node states of the S4P8N cell are retained due to the constructed feedback loops.

(3) We consider the case of reading 1. Before the read operation, bit lines BL and BLN need to be pre-charged to supply voltage. When $\mathrm{WL}=1$, the operation of reading 1 from the S4P8N cell is executed. Since transistors N2, N4, N6, and $\mathrm{N} 8$ are $\mathrm{ON}$, the voltage of BLN decreases while the voltage of $\mathrm{BL}$ does not change. Once the differential sense amplifier detects that the voltage difference between BL and BLN is at a specified constant value, the value stored in the S4P8N cell is successfully read out.

For writing/holding/reading 0 , a similar scenario can be observed. Figure 4 shows the simulation results for normal operations of the S4P8N cell. It can be seen from Fig. 4 that a series of "write 0 ","hold 0", "read 0", "write 1", "hold 1", and "read 1" operations were correctly executed and the written values were correctly retained in the S4P8N cell.

The fault-tolerance principles of the proposed S4P8N cell are described in the following. Here, we still consider the case where the cell stores 1 (i.e., I1 = I3 = 1 and I2 = I4 =0) for illustration purpose. First, we discuss the SNU self-recovery principle of the proposed S4P8N cell.

\section{A. SNU Self-Recovery Principle}

The SNU self-recovery principle is provided according to the state shown in Fig. 2. First, we consider the case where I1 is affected by an SNU. When I1 is temporarily changed to 0 from the original value of 1 due to the SNU, N2 becomes OFF. At this time, the SNU is intercepted by N2. Thus, I2 is not affected $(\mathrm{I} 2=0)$ and $\mathrm{N} 7$ remains OFF. Since I3 is also not affected $(\mathrm{I} 3=1)$, N4 remains ON. At the same time, the fact that I1 temporarily changes to 0 from the original value of 1 can cause $\mathrm{P} 4$ to be $\mathrm{ON}$ and $\mathrm{N} 8$ to be OFF temporarily. As a result, I4 is changed to 1 from the original value of 0 temporarily and N5 is ON. On the other hand, since I2 is not affected $(\mathrm{I} 2=0), \mathrm{P} 1$ remains $\mathrm{ON}$ and $\mathrm{N} 1$ remains $\mathrm{OFF}$, making $\mathrm{I} 1=1$. Therefore, I1 can self-recover from the SNU. Note that, when I3 is affected by an SNU, i.e., I3 is temporarily changed to 0 from 1 , the similar SNU self-recovery principle can be observed.

Next, we consider the case where I 2 is affected by an SNU. When $\mathrm{I} 2$ is temporarily changed to 1 from the original value of 0 due to the SNU, P1 becomes OFF and N1 and N7 become ON. At this time, I4 is not affected by the SNU, it still has the original value $(\mathrm{I} 4=0)$, and hence $\mathrm{N} 3$ and N5 remain OFF and $\mathrm{P} 3$ remains $\mathrm{ON}$. Thus, I1 and $\mathrm{I} 3$ keep their original values (i.e., $\mathrm{I} 1=1$ and $\mathrm{I} 3=1$ ). Since $\mathrm{I} 1=1$ and $\mathrm{I} 3=1, \mathrm{P} 2$ and $\mathrm{P} 4$ become OFF and N2, N4, N6, and N8 become ON. Thus, I2 can self-recover from the SNU. Note that, when I4 is affected by

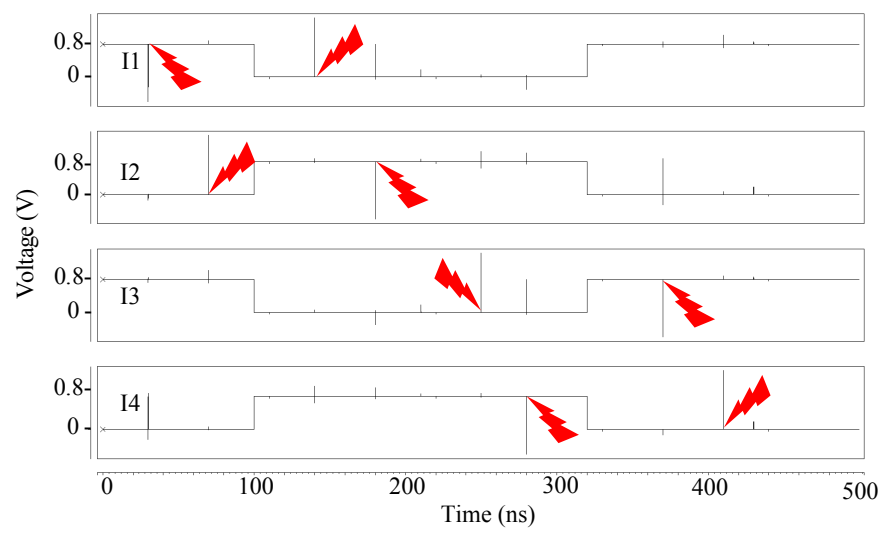

Fig. 5. Simulation results for SNU self-recovery of the proposed S4P8N cell.

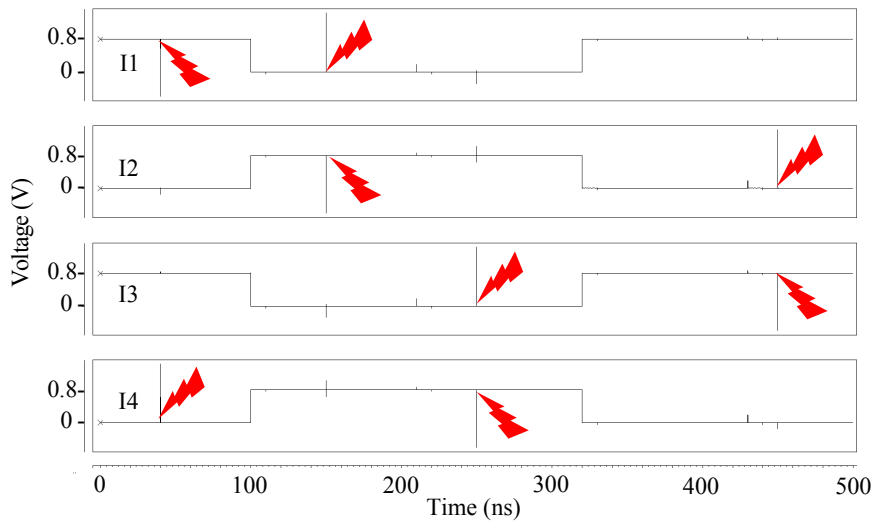

Fig. 6. Simulation results for DNU tolerance of the proposed S4P8N cell.

an SNU, i.e., I4 is temporarily changed to 1 from 0 , the similar SNU self-recovery principle can be observed.

In the case of 0 being stored in the cell, it can be concluded from analysis and simulation that the cell can also self-recover 
from any SNU. We consider the case where I1 is affected by an SNU. When I1 is temporarily changed to 1 from the original value of 0 due to the SNU, P4 becomes OFF and N2 and N8 become ON. At this time, I3 is not affected by the $\mathrm{SNU}$, and it still has the original value $(\mathrm{I} 3=1)$. Hence, N4 and N6 remain $\mathrm{ON}$ and $\mathrm{P} 2$ remains OFF. Thus, I2 and I4 keep their original values (i.e., I2 $=0$ and $\mathrm{I} 4=0$ ). Since $\mathrm{I} 2=0$ and $\mathrm{I} 4=0, \mathrm{P} 1$ and $\mathrm{P} 3$ become $\mathrm{ON}$ and $\mathrm{N} 1, \mathrm{~N} 3, \mathrm{~N} 5$, and $\mathrm{N} 7$ become OFF. Thus, I1 can self-recover from the SNU. Note that, when I3 is affected by an SNU, i.e., I3 is temporarily changed to 1 from 0 , the similar SNU self-recovery principle can be observed.

Next, we consider the case where I 2 is affected by an SNU. When $\mathrm{I} 2$ is temporarily changed to 0 from the original value of 1 due to the SNU, N7 becomes OFF. At this time, the SNU is intercepted by N7. Thus, I3 is not affected (I3 =0) and N4 remains OFF. Since I4 is also not affected (I4=1), N5 remains $\mathrm{ON}$. At the same time, the fact that $\mathrm{I} 2$ temporarily changes to 0 from the original value of 1 can cause $\mathrm{P} 1$ to be $\mathrm{ON}$ and $\mathrm{N} 1$ to be OFF temporarily. As a result, I1 is changed to 1 from the original value of 0 temporarily and $\mathrm{N} 2$ is $\mathrm{ON}$. On the other hand, since $\mathrm{I} 3$ is not affected $(\mathrm{I} 3=0), \mathrm{P} 2$ remains $\mathrm{ON}$ and $\mathrm{N} 6$ remains $\mathrm{OFF}$, making $\mathrm{I} 2=1$. Therefore, $\mathrm{I} 2$ can self-recover from the SNU. Note that, when I4 is affected by an SNU, i.e., I4 is temporarily changed to 0 from 1 , the similar SNU self-recovery principle can be observed.

The simulation results for SNU self-recovery on nodes I1 to I4 of the proposed S4P8N cell are shown in Fig. 5. In the case of 1 being stored $(\mathrm{I} 1=1)$, SNUs were injected to nodes I1 to I4 between 0 and $100 \mathrm{~ns}$ and between 350 and $450 \mathrm{~ns}$, respectively. In the case of 0 being stored $(\mathrm{I} 1=0)$, SNUs were injected to nodes I1 to I4 between 100 and $300 \mathrm{~ns}$, respectively. From Fig. 5, it can be seen that the proposed S4P8N cell can self-recover from all possible SNUs.

\section{B. DNU-Tolerance Principle}

Let us now consider the DNU tolerance principle of the proposed S4P8N cell. The key node-pairs are $<\mathrm{I} 1, \mathrm{I} 2\rangle,<\mathrm{I} 1$, $\mathrm{I} 3>,<\mathrm{I} 1, \mathrm{I} 4>,<\mathrm{I} 2, \mathrm{I} 3>,<\mathrm{I} 2, \mathrm{I} 4>$ and $<\mathrm{I} 3, \mathrm{I} 4>$. We first describe the case where the S4P8N cell stores 1 .

First, we consider the case where $<\mathrm{I} 1$, I4 $>$ is affected by a DNU, i.e., I1 is temporarily changed to 0 from the original value of 1 and I4 is temporarily changed to 1 from the original value of 0 . Thus, $\mathrm{N} 2$ becomes temporarily $\mathrm{OFF}$ and $\mathrm{N} 5$ becomes temporarily ON. Since I2 is not directly affected (I2 $=0$ ), $\mathrm{P} 1$ remains $\mathrm{ON}$ and $\mathrm{N} 1$ remains $\mathrm{OFF}$. As a result, $\mathrm{I} 1=1$, P4 becomes OFF, and N8 becomes ON. Since I3 is also not directly affected $(\mathrm{I} 3=1)$, N4 remains ON. Finally, all nodes and transistors can return to their original states. In other words, $<\mathrm{I} 1, \mathrm{I} 4>$ of the proposed S4P8N cell can self-recover from the DNU.

Second, we consider the case where $<\mathrm{I} 2, \mathrm{I} 3>$ is affected by a DNU, i.e., I2 is temporarily changed to 1 from the original value of 0 and $\mathrm{I} 3$ is temporarily changed to 0 from the original value of 1 . Thus, N4 becomes temporarily OFF and N7 becomes temporarily ON. Since I4 is not directly affected (I4 $=0$ ), $\mathrm{P} 3$ remains $\mathrm{ON}$ and $\mathrm{N} 3$ remains OFF. As a result, $\mathrm{I} 3=1$, P2 becomes OFF, and N6 becomes ON. Since I1 is also not directly affected $(\mathrm{I} 1=1)$, N2 remains ON. Finally, all nodes and transistors can return to their original states. In other words, $<\mathrm{I} 2, \mathrm{I} 3>$ of the proposed S4P8N cell can self-recover from the DNU.

Finally, we consider the case where $<\mathrm{I} 1, \mathrm{I} 2>$ is affected by a DNU, i.e., I1 is temporarily changed to 0 from the original value of 1 and $\mathrm{I} 2$ is temporarily changed to 1 from the original value of 0 . Thus, $\mathrm{N} 2$ becomes temporarily OFF and N7 becomes temporarily $\mathrm{ON}$. When $\mathrm{I} 1$ is changed to $0, \mathrm{P} 4$ is $\mathrm{ON}$ and N8 is OFF. Thus, I4 gets an invalid value (I4=1). As a result, P3 becomes OFF and N3 becomes ON. Since N7 is ON as mentioned above, I3 gets an invalid value $(\mathrm{I} 3=0)$. Consequently, all nodes and transistors cannot return to their original states. In other words, the proposed $\mathrm{S} 4 \mathrm{P} 8 \mathrm{~N}$ cell cannot tolerate the DNU on $<\mathrm{I} 1, \mathrm{I} 2>$. Note that, when the other node pairs of the cell suffer from a DNU, similar results can be observed.

To summarize, when the proposed S4P8N cell stores 1, node pairs $<$ I2, I3 $>$ and $<$ I1, I4 $>$ of the cell can self-recover from a DNU, but the other node pairs of the cell cannot self-recover from a DNU. Next, we discuss the case of 0 being stored in the proposed S4P8N cell.

First, we consider the case where $<\mathrm{I} 1, \mathrm{I} 2>$ is affected by a DNU, i.e., I1 is temporarily changed to 1 from the original value of 0 and $\mathrm{I} 2$ is temporarily changed to 0 from the original value of 1 . Thus, $\mathrm{N} 2$ becomes temporarily $\mathrm{ON}$ and $\mathrm{N} 7$ becomes temporarily OFF. Since I3 is not directly affected (I3 $=0), \mathrm{P} 2$ remains $\mathrm{ON}$ and $\mathrm{N} 6$ remains OFF. As a result, $\mathrm{I} 2=1$, P1 becomes OFF, and N1 becomes ON. Since I4 is also not directly affected $(\mathrm{I} 4=1)$, N5 remains ON. Finally, all nodes and transistors can return to their original states. In other words, $<\mathrm{I} 1$, I2 $>$ of the proposed S4P8N cell can self-recover from the DNU.

Second, we consider the case where $<\mathrm{I} 3, \mathrm{I} 4>$ is affected by a DNU, i.e., I3 is temporarily changed to 1 from the original value of 0 and $\mathrm{I} 4$ is temporarily changed to 0 from the original value of 1 . Thus, N4 becomes temporarily $\mathrm{ON}$ and $\mathrm{N} 5$ becomes temporarily OFF. Since I1 is not directly affected (I1 $=0$ ), $\mathrm{P} 4$ remains $\mathrm{ON}$ and $\mathrm{N} 8$ remains OFF. As a result, $\mathrm{I} 4=1$, P3 becomes OFF, and N3 becomes ON. Since I2 is also not directly affected $(\mathrm{I} 2=1)$, N7 remains ON. Finally, all nodes and transistors can return to their original states. In other words, $<\mathrm{I} 3, \mathrm{I} 4>$ of the proposed $\mathrm{S} 4 \mathrm{P} 8 \mathrm{~N}$ cell can self-recover from the DNU.

Finally, we consider the case where $<\mathrm{I} 2, \mathrm{I} 3>$ is affected by a $\mathrm{DNU}$, i.e., $\mathrm{I} 2$ is temporarily changed to 0 from the original value of 1 and $\mathrm{I} 3$ is temporarily changed to 1 from the original value of 0 . Thus, $\mathrm{N} 7$ becomes temporarily $\mathrm{OFF}$ and $\mathrm{N} 4$ becomes temporarily $\mathrm{ON}$. When $\mathrm{I} 2$ is changed to $0, \mathrm{P} 1$ is $\mathrm{ON}$ and $\mathrm{N} 1$ is OFF. Thus, $\mathrm{I} 1$ gets an invalid value $(\mathrm{I} 1=1)$. As a result, $\mathrm{P} 4$ becomes OFF and N8 becomes ON. Since N4 is ON as mentioned above, I4 gets an invalid value $(\mathrm{I} 4=0)$. Finally, all nodes and transistors cannot return to their original states. In other words, the proposed S4P8N cell cannot tolerate the $\mathrm{DNU}$ on $<\mathrm{I} 2, \mathrm{I} 3>$. Note that, when the other node pairs of the cell suffer from a DNU, similar results can be observed.

To summarize, when the proposed S4P8N cell stores 0 , node pairs $<$ I1, I2 $>$ and $<$ I3, I $4>$ of the cell can self-recover from a DNU, but the other node pairs of the cell cannot self-recover from a DNU. 
Figure 6 shows the simulation results for DNU self-recovery of node pairs $<\mathrm{I} 1, \mathrm{I} 4>,<\mathrm{I} 1, \mathrm{I} 2>,<\mathrm{I} 3, \mathrm{I} 4>$, and $<\mathrm{I} 2, \mathrm{I} 3>$ of the proposed S4P8N cell. In the case of 1 being stored $(\mathrm{I} 1=1)$, DNUs were injected to node pairs $<\mathrm{I} 1, \mathrm{I} 4>$ and $<\mathrm{I} 2$, I3 $>$ between 0 and $100 \mathrm{~ns}$ and between 400 to $500 \mathrm{~ns}$, respectively. In the case of 0 being stored ( $\mathrm{I} 1=0)$, DNUs were injected to node pairs $<\mathrm{I} 1, \mathrm{I} 2>$ and $<\mathrm{I} 3$, I4 $>$ between 100 and 300 ns, respectively. From Fig. 6, it can be concluded that the proposed S4P8N cell can self-recover from DNUs on node pairs $<\mathrm{I} 1, \mathrm{I} 4>$ and $<\mathrm{I} 2, \mathrm{I} 3>$ when storing 1 and the proposed S4P8N cell can self-recover from DNUs on node pairs $<$ I1, I2 $>$ and $<\mathrm{I} 3, \mathrm{I} 4>$ when storing 0 (totally four node-pairs are DNU-self-recoverable).

\section{PROPOSED S8P4N SRAM CELL}

Figure 7 shows the schematic of the proposed S8P4N cell. It can be seen from Fig. 7 that the S8P4N cell consists of 16 transistors, i.e., PMOS transistors $\mathrm{P} 1$ to $\mathrm{P} 8$ and NMOS transistors $\mathrm{N} 1$ to $\mathrm{N} 8$. The storage part of the cell has 8 PMOS transistors and 4 NMOS transistors, i.e., transistors P1 to P8 and N1 to N4. Transistors N5 to N8 are used for access operations and their gate terminals are connected to word-line WL. In the proposed S8P4N cell, I1 to I4 are internal nodes and they are connected to bit lines BL and BLN through access transistors N5 to N8, respectively. Figure 8 shows the layout of the proposed S8P4N cell. When $\mathrm{WL}=1$, the access transistors are $\mathrm{ON}$, allowing write/read access operations to be executed. When $\mathrm{WL}=0$, the access transistors are OFF, and the cell retains the stored value. Note that, the normal operations of the proposed S8P4N cell are similar to those of the proposed S4P8N cell. So, detailed descriptions are omitted here.

Figure 9 shows the simulation results for normal operations of the proposed S8P4N cell. It can be seen that the cell can correctly perform the "write 0 , hold 0 , read 0 , write 1 , hold 1 , and read 1 " operations.

The fault tolerance principles of the proposed S8P4N cell are described as follows. For illustration purpose, we still consider the case of 1 being stored in the cell (i.e., $\mathrm{I} 1=\mathrm{I} 3=1$ and $\mathrm{I} 2=\mathrm{I} 4=0$ ).

\section{A. SNU Self-Recovery Principle}

The SNU self-recovery principle is provided according to the state shown in Fig. 7. First, we consider the case where I1 is affected due to the SNU, i.e., I1 is temporarily changed to 0 from 1. At this time, since I1 is flipped, P2 and P8 become ON and N4 becomes OFF. I3 is not affected, i.e., I3 = 1, and hence $\mathrm{P} 4$ and $\mathrm{P} 6$ remain OFF and $\mathrm{N} 2$ remains ON. Thus, I2 is not affected $(\mathrm{I} 2=0)$, and $\mathrm{I} 4$ still has its previous correct value of 0 . Since $\mathrm{I} 2=0$ and $\mathrm{I} 4=0, \mathrm{P} 1$ and $\mathrm{P} 5$ remain $\mathrm{ON}$ and $\mathrm{N} 1$ remains OFF. Thus, $\mathrm{I} 1$ can return to the original correct state, i.e., $\mathrm{I} 1=$ 1. Therefore, I1 can self-recover from the SNU. Note that, when I3 is affected due to the SNU, i.e., I3 is temporarily changed to 0 from 1 , a similar SNU self-recovery principle can be observed.

Next, we consider the case where 12 is affected due to the SNU, i.e., I 2 is temporarily changed to 1 from 0 . At this time,
$\mathrm{N} 1$ becomes $\mathrm{ON}$ and $\mathrm{P} 1$ and $\mathrm{P} 7$ become OFF. Thus, I1 is temporarily changed to 0 from 1 , and $\mathrm{P} 2$ and $\mathrm{P} 8$ become temporarily ON and N4 becomes temporarily OFF. Since I3 is not affected by the SNU (it still has the original value of 1), P4

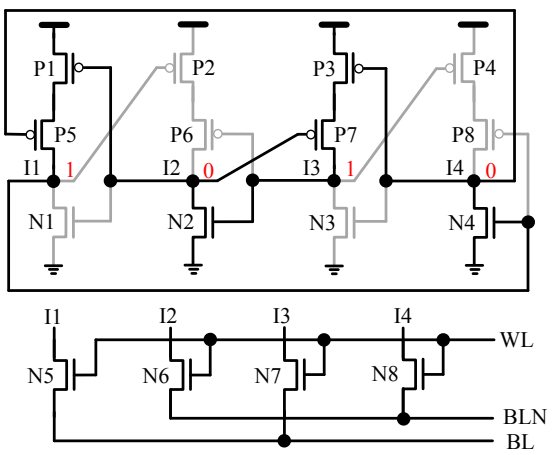

Fig. 7. Schematic of the proposed S8P4N cell.

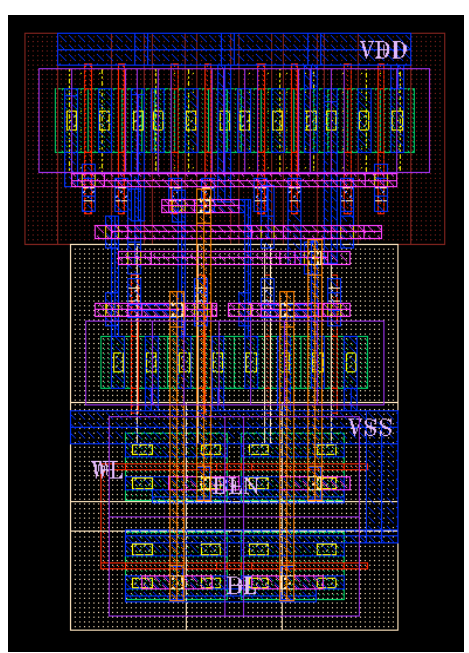

Fig. 8. Layout of the proposed S8P4N cell.

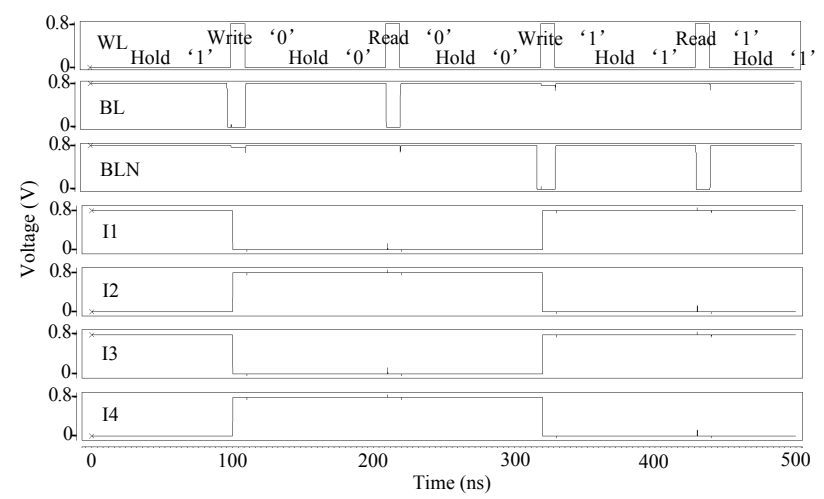

Fig. 9. Simulation results for normal operations of the proposed S8P4N cell.

and $\mathrm{P} 6$ remain $\mathrm{OFF}$ and $\mathrm{N} 2$ remains $\mathrm{ON}$. Thus, $\mathrm{I} 2$ can return to its original correct value of 0 and $\mathrm{I} 4$ still has its previous correct value of 0 . Since $\mathrm{I} 2=0$ and $\mathrm{I} 4=0, \mathrm{P} 1$ and $\mathrm{P} 5$ can return to their $\mathrm{ON}$ state and $\mathrm{N} 1$ can return to its OFF state. Therefore, I1 can return to its original value. In other words, the cell can self-recover from the SNU. Note that, when I4 is affected due to the SNU, i.e., I4 is temporarily changed to 1 from 0 , a similar SNU self-recovery principle can be observed. In the case of 0 being stored in the cell, it can be concluded 
from analysis and simulation that the cell can also self-recover from any SNU. We consider the case where I1 is affected due to the SNU, i.e., I1 is temporarily changed to 1 from 0 . At this time, N4 becomes ON and P2 and P8 become OFF. Thus, I4 is temporarily changed to 0 from 1 , and P3 and P5 become temporarily $\mathrm{ON}$ and $\mathrm{N} 3$ becomes temporarily OFF. Since I 2 is not affected by the SNU (still has the original correct value of 1), $\mathrm{P} 1$ and $\mathrm{P} 7$ remain OFF and $\mathrm{N} 1$ remains ON. Thus, I1 can return to its original correct value of 0 and $\mathrm{I} 3$ still has its previous correct value of 0 . Since $\mathrm{I} 1=0$ and $\mathrm{I} 3=0, \mathrm{P} 4$ and $\mathrm{P} 8$ return to their $\mathrm{ON}$ state and $\mathrm{N} 4$ returns to its OFF state. Therefore, I4 can return to its original correct value. In other words, the cell can self-recover from the SNU. Note that, when I3 is affected due to the SNU, i.e., I3 is temporarily changed to 1 from 0 , a similar SNU self-recovery principle can be observed.

Next, we consider the case where 12 is affected due to the $\mathrm{SNU}$, i.e., $\mathrm{I} 2$ is temporarily changed to 0 from 1 . At this time, since $\mathrm{I} 2$ is temporarily flipped, $\mathrm{P} 1$ and $\mathrm{P} 7$ become $\mathrm{ON}$ and $\mathrm{N} 1$ becomes OFF. I4 is not affected, i.e., I4 = 1, and hence P3 and $\mathrm{P} 5$ remain OFF and N3 remains ON. Thus, I3 is not affected $(\mathrm{I} 3=0)$, and $\mathrm{I} 1$ still has its previous correct value of 0 . Since $\mathrm{I} 1=0$ and $\mathrm{I} 3=0, \mathrm{P} 2$ and $\mathrm{P} 6$ remain $\mathrm{ON}$ and $\mathrm{N} 2$ remains $\mathrm{OFF}$. Thus, $\mathrm{I} 2$ can return to the original correct state, i.e., $\mathrm{I} 2=1$. Therefore, I2 can self-recover from the SNU. Note that, when I4 is affected due to the SNU, i.e., I4 is temporarily changed to 0 from 1, a similar SNU self-recovery principle can be observed. To summarize, the S8P4N cell can provide a complete SNU self-recoverability.

Figure 10 shows the simulation results for SNU self-recovery of nodes I1, I2, I3, and I4 of the proposed S8P4N cell. As shown in Fig. 10, in the case of 1 being stored, an SNU was injected to I1, I2, I3, and I4 between 0 and $100 \mathrm{ns,}$ and between 350 and $450 \mathrm{~ns}$, respectively. In the case of 0 being stored, an SNU was injected to I1, I2, I3, and I4 between 100 and $300 \mathrm{~ns}$, respectively. As can be seen, all nodes of the S8P4N cell can self-recover from SNUs.

\section{B. DNU-Tolerance Principle}

Let us consider the DNU self-recovery principle of the proposed S8P4N cell. The cell has six key node-pairs, i.e., $<\mathrm{I} 1$, $\mathrm{I} 2>,<\mathrm{I} 1, \mathrm{I} 3>,<\mathrm{I} 1, \mathrm{I} 4>,<\mathrm{I} 2, \mathrm{I} 3>,<\mathrm{I} 2, \mathrm{I} 4>$ and $<\mathrm{I} 3, \mathrm{I} 4>$. Here we discuss the case of 1 being stored.

First, we consider the case where $<\mathrm{I} 1, \mathrm{I} 2>$ is affected by a DNU, i.e., I1 is temporarily changed to 0 and I 2 is temporarily changed to 1 . Since $\mathrm{I} 1=0$ and $\mathrm{I} 2=1, \mathrm{P} 2, \mathrm{P} 8$ and $\mathrm{N} 1$ become $\mathrm{ON}$, and P1, P7 and N4 become OFF. Since I3 is not affected by the DNU (I3 still has its previous correct value of 1), P4 and $\mathrm{P} 6$ remain OFF and $\mathrm{N} 2$ remains ON. Therefore, I4 still has its previous correct value of $0, \mathrm{P} 5$ remains $\mathrm{ON}$, and $\mathrm{I} 2$ can return to its original correct value of 0 . Thus, $\mathrm{P} 1$ and $\mathrm{P} 7$ return to their ON state, and N1 returns to their OFF state, so I1 can return to its original correct value of 1 . In other words, $<\mathrm{I} 1$, I2> can self-recover from the DNU.

Second, we consider the case where $<\mathrm{I} 3, \mathrm{I} 4>$ is affected by a DNU, i.e., I3 is temporarily changed to 0 and I4 is temporarily changed to 1 . At this time, P4, P6 and N3 become $\mathrm{ON}$, and P3, P5 and N2 become OFF. Since I1 is not affected by the DNU, I1 still has its previous correct value of 1, and P2 and $\mathrm{P} 8$ remain $\mathrm{OFF}$ and $\mathrm{N} 4$ remains $\mathrm{ON}$. Therefore, I2 still has its previous correct value of $0, \mathrm{P} 7$ remains $\mathrm{ON}$, and $\mathrm{I} 4$ can return to its original correct value of 0 . Thus, P3 and P5 return to their ON state, and N3 returns to its OFF state, so I3 can return to its original correct value of 1 . In other words, $<\mathrm{I} 3$, I4> can self-recover from the DNU.

Finally, we consider the case where $<\mathrm{I} 1, \mathrm{I} 3>$ is affected by a DNU, i.e., I1 and I3 are temporarily changed to 0 . Thus, N2 and N4 become OFF and P2, P4, P6, and P8 become ON, so that the values of I2 and I4 are flipped. In other words, all nodes of the cell are flipped, and $<$ I1, I3 $>$ cannot self-recover from the DNU. Note that for $<\mathrm{I} 1, \mathrm{I} 4>,<\mathrm{I} 2, \mathrm{I} 3>$, and $<\mathrm{I} 2, \mathrm{I} 4>$, similar results can be observed.

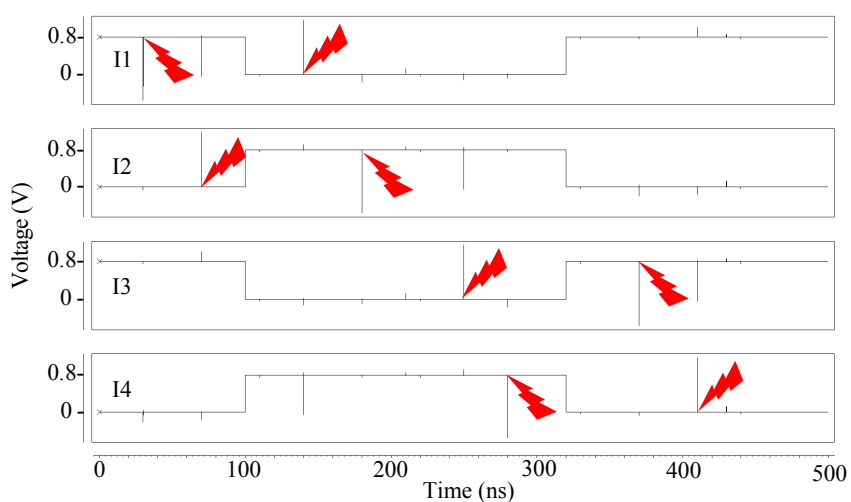

Fig. 10. Simulation results for SNU self-recovery of the proposed S8P4N cell.

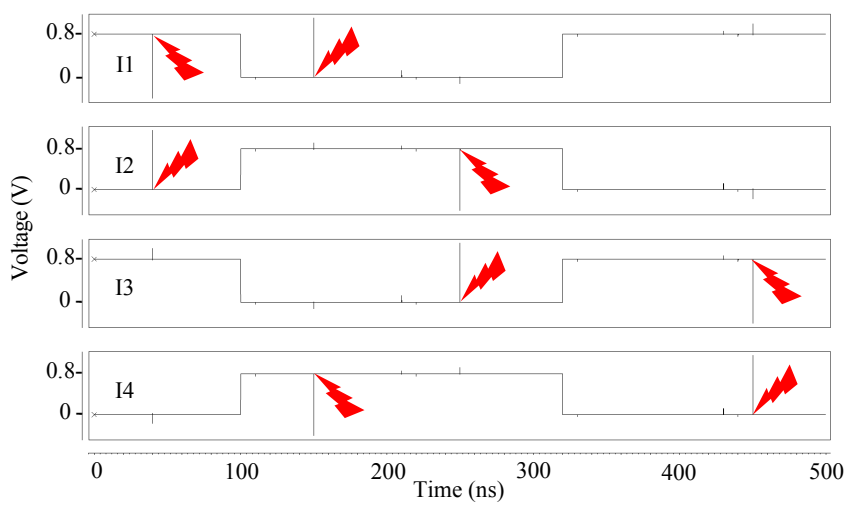

Fig. 11. Simulation results for DNU self-recovery of the proposed S8P4N cell.

To summarize, for the proposed S8P4N cell, in the case of 1 being stored, node pairs $<\mathrm{I} 1, \mathrm{I} 2>$ and $<\mathrm{I} 3$, I4 $>$ can self-recover from DNUs, but the other node pairs cannot self-recover from DNUs. Next, we discuss the case of 0 being stored for the cell.

First, we consider the case where $<\mathrm{I} 1, \mathrm{I} 4>$ is affected by a DNU, i.e., I1 is temporarily changed to 1 and I4 is temporarily changed to 0 , and hence P3, P5, and N4 become ON and P2, P8, and N3 become OFF. Since I2 is not affected by the DNU, I2 still has its previous correct value of 1 , and P1 and P7 remain $\mathrm{OFF}$ and $\mathrm{N} 1$ remains $\mathrm{ON}$. Thus, $\mathrm{I} 3$ still has its previous correct value 0 and $\mathrm{I} 1$ can return to its original correct value of 0 . At this time, $\mathrm{N} 2$ and $\mathrm{N} 4$ remain $\mathrm{OFF}$ and $\mathrm{P} 2, \mathrm{P} 4, \mathrm{P} 6$, and $\mathrm{P} 8$ remain $\mathrm{ON}$, so that $\mathrm{I} 4$ can return to its original correct value of 1. In other words, $<\mathrm{I} 1, \mathrm{I} 4>$ can self-recover from the DNU. 
Second, we consider the case where $<\mathrm{I} 2, \mathrm{I} 3>$ is affected by a DNU, i.e., I2 is temporarily changed to 0 and $\mathrm{I} 3$ is temporarily changed to 1 , and hence $\mathrm{P} 1, \mathrm{P} 7$, and $\mathrm{N} 2$ become $\mathrm{ON}$ and P4, P6, and N1 become OFF. Since I4 is not affected by the DNU, I4 still has its previous correct value of 1, and P3 and $\mathrm{P} 5$ remain OFF and $\mathrm{N} 3$ remains ON. Thus, I1 still has its previous correct value of 0 and $\mathrm{I} 3$ can return to its original correct value of 0 . At this time, $\mathrm{N} 2$ and $\mathrm{N} 4$ remain OFF and $\mathrm{P} 2$, P4, P6, and P8 remain ON, so that $\mathrm{I} 2$ can return to its original correct value of 1 . In other words, $<\mathrm{I} 2, \mathrm{I} 3>$ can self-recover from the DNU.

Finally, we consider the case where $<\mathrm{I} 1, \mathrm{I} 2>$ is affected by a DNU, i.e., I1 is temporarily changed to 1 and $\mathrm{I} 2$ is temporarily changed to 0 . At this time, $\mathrm{P} 2, \mathrm{P} 8$ and $\mathrm{N} 1$ become OFF and $\mathrm{P} 1$, $\mathrm{P} 7$, and $\mathrm{N} 4$ become ON, and hence I4 is flipped to 0 . Thus, $\mathrm{P} 3$ and P5 become ON and N3 becomes OFF. Since P3 and P7 become ON and $\mathrm{N} 3$ becomes OFF, I3 is flipped to 0 . In other words, all nodes of the cell are flipped. Therefore, $<$ I1, I2 $>$ cannot self-recover from the DNU. Note that for $<\mathrm{I} 1, \mathrm{I} 3>,<\mathrm{I} 2$, I4 $>$, and $<\mathrm{I} 3$, I4 $>$, similar DNU self-recovery results can be observed. To summarize, for the proposed S8P4N cell, in the case of 0 being stored, node pairs $<$ I1, I $4>$ and $<$ I2, I $3>$ can self-recover from a DNU, but the other node pairs cannot self-recover from a DNU.

Figure 11 shows the simulation results for DNU self-recovery of node pairs $<\mathrm{I} 1, \mathrm{I} 2>,<\mathrm{I} 1, \mathrm{I} 4>,<\mathrm{I} 2, \mathrm{I} 3>$, and $<\mathrm{I}$, I4 $>$ of the proposed S8P4N cell. In the case of 1 being stored (I1 $=1$ ), DNUs were injected to node pairs $<\mathrm{I} 1, \mathrm{I} 2>$ and $<\mathrm{I} 3$, I $>>$ between 0 and $100 \mathrm{~ns}$ and between 400 to $500 \mathrm{~ns}$, respectively. In the case of 0 being stored (I1 $=0)$, DNUs were injected to node pairs $<\mathrm{I} 1, \mathrm{I} 4>$ and $<\mathrm{I} 2, \mathrm{I} 3>$ between 100 and 300 ns, respectively. From Fig. 11, we can conclude that the proposed S8P4N cell can self-recover from DNUs on node pairs $<\mathrm{I} 1, \mathrm{I} 2>$ and $<\mathrm{I} 3$, I $4>$ when storing 1 and can self-recover from DNUs on node pairs $<$ I1, I4 $>$ and $<$ I2, I3 $>$ when storing 0 (totally four node-pairs are DNU-self-recoverable).

TABLE I

SUMMARY OF NODE-UPSET TOLERANCE OF THE PROPOSED SRAMS.

\begin{tabular}{|c|c|c|c|c|}
\hline & \multicolumn{2}{|c|}{ S4P8N } & \multicolumn{2}{|c|}{ S8P4N } \\
\hline & When $\mathrm{I} 1=1$ & When $\mathrm{I} 1=0$ & When $I 1=1$ & When $\mathrm{I} 1=0$ \\
\hline SRN & $\begin{array}{c}\mathrm{I} 1, \mathrm{I} 2, \mathrm{I} 3 \\
\mathrm{I} 4 .\end{array}$ & $\begin{array}{c}\mathrm{I} 1, \mathrm{I} 2, \mathrm{I} 3 \\
\mathrm{I} 4 .\end{array}$ & $\begin{array}{c}\mathrm{I} 1, \mathrm{I} 2, \mathrm{I} 3 \\
\mathrm{I} 4 .\end{array}$ & $\begin{array}{c}\mathrm{I} 1, \mathrm{I} 2, \mathrm{I} 3 \\
\mathrm{I} 4 .\end{array}$ \\
\hline DHP & $\begin{array}{l}<\mathrm{I} 1, \mathrm{I} 4> \\
<\mathrm{I} 2, \mathrm{I} 3>\end{array}$ & $\begin{array}{l}<\mathrm{I} 1, \mathrm{I} 2> \\
<\mathrm{I} 3, \mathrm{I} 4>\end{array}$ & $\begin{array}{l}<\mathrm{I} 1, \mathrm{I} 2> \\
<\mathrm{I} 3, \mathrm{I} 4>\end{array}$ & $\begin{array}{l}<\mathrm{I} 1, \mathrm{I} 4> \\
<\mathrm{I} 2, \mathrm{I} 3>\end{array}$ \\
\hline DNHP & $\begin{array}{l}<\mathrm{I} 1, \mathrm{I} 2> \\
<\mathrm{I} 1, \mathrm{I} 3> \\
<\mathrm{I} 2, \mathrm{I} 4> \\
<\mathrm{I} 3, \mathrm{I} 4>\end{array}$ & $\begin{array}{l}<\mathrm{I} 1, \mathrm{I} 3> \\
<\mathrm{I} 1, \mathrm{I} 4> \\
<\mathrm{I} 2, \mathrm{I} 3> \\
<\mathrm{I} 2, \mathrm{I} 4>\end{array}$ & $\begin{array}{l}<\mathrm{I} 1, \mathrm{I} 3> \\
<\mathrm{I} 1, \mathrm{I} 4> \\
<\mathrm{I} 2, \mathrm{I} 3> \\
<\mathrm{I} 2, \mathrm{I} 4>\end{array}$ & $\begin{array}{l}<\mathrm{I} 1, \mathrm{I} 2> \\
<\mathrm{I} 1, \mathrm{I} 3> \\
<\mathrm{I} 2, \mathrm{I} 4> \\
<\mathrm{I} 3, \mathrm{I} 4>\end{array}$ \\
\hline
\end{tabular}

In summary, the proposed SRAM cells can provide complete self-recovery from all possible SNUs and partial recovery from DNUs. Table I shows a summary of node-upset tolerance of the proposed SRAMs. In Table I, SRN means SNU Recoverable single Nodes, DHP means DNU-Hardened node Pairs, and DNHP means DNU-Not-Hardened node Pairs. It can be seen from Table I that all single nodes of the proposed SRAMs can self-recover from all possible SNUs and the DNU-hardened node pairs of each proposed SRAM cell is up to 4 .

\section{COMPARISON AND EVAluation RESUlts}

To make a fair comparison with the state-of-the-art SRAM cells described in Section I, the same simulation conditions and technology node (22nm CMOS) as those described in the above section were used. Table II shows the reliability and overhead comparison results among the unhardened/hardened SRAMs in terms of $S N U$ recoverability (SNUR), number of DHP (\#DHP), average power dissipation (dynamic and static) that is measured with the method in [22], silicon area that is measured with the method in [30], read access time (RAT), and write access time (WAT). Note that, the hold operations consume static power, the read/write operations consume dynamic power and the operations are shown in Figs. 4 and 9. The percentages of reduced costs (PRCs) of the proposed cells compared with the other cells were calculated using the method in [28] and are also shown in Table II.

Let us first discuss about the reliability comparison. It can be seen from Table II that, the proposed S4P8N and S8P4N cells, and the Lin12T, RH12T, DNUCTM, and DNUSRM cells can provide complete SNU self-recoverability from all possible SNUs, while the other cells cannot provide complete SNU self-recoverability since any of them has at least one node that cannot self-recover from an SNU. Regarding \#DHNPs, the 6T and NASA13T cells have no DHNP, and the We-Quatro, PS10T, Lin12T, RH12T, DNUCTM, and DNUSRM cells have two or more than two DHNPs. Clearly, except the DNUCTM and DNUSRM cells, only the proposed S4P8N and S8P4N cells have the maximum number of DHNPs which is 4. Although the DNUCTM and DNUSRM cells have up to 16 DHNPs, they require large power dissipation and silicon area overhead. This point will be discussed in the following. To summarize, the proposed cells achieve a better balance between reliability and overhead.

For power and area, it can be seen from Table II that the $6 \mathrm{~T}$ cell has the smallest power and area due to the use of only 6 transistors. Generally, a cell having a few transistors has a small area. It can be seen from Table II that the proposed cells have to use extra transistors/area to ensure the self-recoverability from all possible SNUs and a part of DNUs as well as optimized speed and power. However, the proposed S4P8N and S8P4N cells have smaller power compared with the other hardened cells. Note that, the NASA13T consumes the largest power mainly due to the large current competition in its feedback loops and the use of extra access transistors.

For RATs and WATs, it can be seen from Table II that the $6 \mathrm{~T}$ cell has the smallest WAT. This is mainly because the cell has less current competition when writing a value. Conversely, the QCCM10T has the largest WAT due to more current competition when writing a value. It also can be seen from Table II that the proposed S4P8N cell has a comparable WAT and RAT compared with the other hardened cells. The proposed S8P4N has a smaller WAT and RAT than most of the other hardened cells. This is mainly because the cell has less current competition during access operations through parallel access transistors. Note that the $\mathrm{S} 8 \mathrm{P} 4 \mathrm{~N}$ cell has more PMOS transistors and we used smaller transistor sizes for PMOS transistors to achieve better performance compared to the S4P8N cell. The NASA13T has the largest RAT due to its 
special read operation (slow current flow through read transistors). Indeed, the intrinsic charge/discharge operations of cell-nodes through access transistors can affect RATs and WATs. Therefore, the better reliability and optimized overhead of the proposed S4P8N and S8P4N cells are effectively balanced.

Let us now discuss the PRCs. For the sake of brevity, only the average PRCs are discussed. For the proposed S4P8N cell, compared with other hardened cells, the average PRCs of the nano-scale technologies [30-31]. Figure 12 shows the results of PVT variation impacts on power, WAT, and RAT for SRAM cells. Note that, the normal temperature was set to $25^{\circ} \mathrm{C}$ and the temperature was ranged from $-25^{\circ} \mathrm{C}$ to $125^{\circ} \mathrm{C}$. The normal supply voltage was set to $0.8 \mathrm{~V}$ and the supply voltage variation was ranged from $0.65 \mathrm{~V}$ to $0.95 \mathrm{~V}$. The threshold-voltage increment was ranged from $0.01 \mathrm{~V}$ to $0.06 \mathrm{~V}$.

Fig. 12-(a), (b), and (c) show the impact of temperature variations on power, WAT, and RAT. It is clear that as the

TABLE II

RELIABILITY AND OVERHEAD COMPARISON RESULTS AMONG THE UNHARDENED AND HARDENED SRAMS UNDER 22NM CMOS TECHNOLOGY NODE.

\begin{tabular}{|c|c|c|c|c|c|c|c|c|c|c|c|c|c|c|}
\hline & \multirow{3}{*}{ SNUR } & \multirow{3}{*}{ \#DHP } & \multirow{3}{*}{$\begin{array}{c}\text { Power } \\
\text { (nW) }\end{array}$} & \multirow{3}{*}{$\begin{array}{l}10^{-3} \times \\
\text { Area } \\
\left(\mathrm{nm}^{2}\right)\end{array}$} & \multirow{3}{*}{$\begin{array}{l}\text { RAT } \\
\text { (ps) }\end{array}$} & \multirow{3}{*}{$\begin{array}{c}\text { WAT } \\
\text { (ps) }\end{array}$} & \multicolumn{8}{|c|}{ PRC (\%) } \\
\hline & & & & & & & \multicolumn{2}{|c|}{ Power } & \multicolumn{2}{|c|}{ Area } & \multicolumn{2}{|c|}{ RAT } & \multicolumn{2}{|c|}{ WAT } \\
\hline & & & & & & & $4 \mathrm{P} 8 \mathrm{~N}$ & $8 \mathrm{P} 4 \mathrm{~N}$ & $4 \mathrm{P} 8 \mathrm{~N}$ & $8 \mathrm{P} 4 \mathrm{~N}$ & $4 \mathrm{P} 8 \mathrm{~N}$ & $8 \mathrm{P} 4 \mathrm{~N}$ & $4 \mathrm{P} 8 \mathrm{~N}$ & $8 \mathrm{P} 4 \mathrm{~N}$ \\
\hline QCCM10T [12] & $x$ & 1 & 11.45 & 7.79 & 18.20 & 23.21 & 25.33 & 19.13 & -62.64 & -36.71 & 1.48 & 28.90 & 77.64 & 84.19 \\
\hline QCCM12T [12] & $x$ & 1 & 10.43 & 8.71 & 12.99 & 4.22 & 18.02 & 11.22 & -45.46 & -22.27 & -38.03 & 0.38 & -22.99 & 13.03 \\
\hline QUCCE10T [14] & $x$ & 1 & 10.08 & 6.16 & 36.36 & 6.29 & 15.18 & 8.13 & -105.68 & -72.89 & 50.69 & 64.41 & 17.49 & 41.65 \\
\hline QUCCE12T [14] & $x$ & 1 & 10.43 & 8.71 & 13.02 & 4.31 & 18.02 & 11.22 & -45.46 & -22.27 & -37.71 & 0.61 & -20.42 & 14.85 \\
\hline We-Quatro [15] & $x$ & 2 & 10.43 & 8.71 & 12.99 & 4.38 & 18.02 & 11.22 & -45.46 & -22.27 & -38.03 & 0.38 & -18.49 & 16.21 \\
\hline PS10T [16] & $\times$ & 2 & 10.14 & 7.30 & 25.79 & 5.14 & 15.68 & 8.68 & -73.56 & -45.89 & 30.48 & 49.83 & -0.97 & 28.60 \\
\hline DNUSRM [19] & $\sqrt{ }$ & 16 & 20.86 & 17.42 & 6.63 & 4.71 & 59.01 & 55.61 & 27.27 & 38.86 & -170.44 & -95.17 & -10.19 & 22.08 \\
\hline S4P8N (Proposed) & $\sqrt{ }$ & 4 & 8.55 & 12.67 & 17.93 & 5.19 & 26.72 & - & -41.06 & - & -10.50 & - & 0.27 & - \\
\hline S8P4N (Proposed) & $\sqrt{ }$ & 4 & 9.26 & 10.65 & 12.94 & 3.67 & - & 20.63 & - & -18.57 & - & 20.25 & - & 29.48 \\
\hline
\end{tabular}

power dissipation, silicon area, RAT, and WAT are $26.72 \%$, $-41.06 \%,-10.50 \%$, and $0.27 \%$, respectively. It means that the power dissipation of the proposed $\mathrm{S} 4 \mathrm{P} 8 \mathrm{~N}$ cell is reduced approximately by $27 \%$ on average mainly at the cost of silicon area. For the proposed S8P4N cell, compared with the state-of-the-art hardened cells, the average PRCs of the power dissipation, silicon area, RAT, and WAT are $20.63 \%,-18.57 \%$, $20.25 \%$, and $29.48 \%$, respectively. It means that the power dissipation, RAT, and WAT of the proposed S8P4N cell are reduced approximately by $21 \%, 20 \%$, and $29 \%$ on average, at the cost of moderate silicon area.

To summarize, the fault-tolerance ability of the proposed S4P8N and S8P4N cells are achieved mainly at the cost of extra indispensable silicon area compared with the existing state-of-the-art hardened cells. Moreover, compared with most of the existing state-of-the-art hardened cells, the proposed S4P8N cell has a low overhead especially in terms of power dissipation, and the proposed S4P8N cell has a lower overhead especially in terms of power dissipation, RAT, and WAT.

The process, voltage and temperature $(P V T)$ variations can seriously impact the performance of SRAM cells, and SRAM cells are more sensitive to PVT variations in advanced temperature increases, the SRAM cells need to consume more power, WAT, and RAT. It can be seen from Fig. 12-(a) that the DNUSRM and DNUCTM are more sensitive to temperature variations on power, mainly due to the large current competition in their feedback loops and the use of extra access transistors. However, the 6T and S4P8N are less sensitive to temperature variations on power. It can be seen from Fig. 12-(b) that the QUCCE10T is more sensitive to temperature variations on WAT, and the NASA13T cell is less sensitive to temperature variations on WAT. It can be seen from Fig. 12-(c) that the NASA13T is more sensitive to temperature variations on RAT, mainly due to its more decreased carrier mobility when the temperature is rising. Otherwise, the temperature variations have a low impact on the RAT of the cells, such as DNUSRM, QCCM12T, S4P8N, and S8P4N.

Fig. 12-(d), (e), and (f) show the impact of supply voltage variations on power, WAT, and RAT. It is clear that as the supply voltage increases, large supply voltage can lead to large power dissipation of SRAM cells but can reduce access time. It can be seen from Fig. 12-(d) that the DNUSRM cell is the most sensitive to supply voltage variations on power, but the $6 \mathrm{~T}$ is less sensitive to supply voltage variations on power 
mainly due to the use of only 6 transistors. It can be seen from Fig. 12-(e) that the QCCM10T is the most sensitive to supply voltage variations on WAT, but the variations have a low impact on the WAT of the cells, such as QCCM12T, S4P8N and S8P4N. It can be seen from Fig. 12-(f) that the NASA13T cell is the most sensitive to supply voltage variations on RAT. This is mainly due to its special read operation. Otherwise, the supply voltage variations have a low impact on the RAT of the cells, such as DNUSRM, QCCM12T, DNUCTM, S4P8N, and S8P4N.
Fig. 12-(g), (h), and (i) show the impact of threshold voltage variations on power, WAT, and RAT. It is clear that as the threshold voltage increases, large threshold voltage can decrease power dissipation of SRAM cells but can increase access time. It can be seen from Fig 12-(g) that the DNUSRM is the most sensitive to threshold voltage variation on power mainly due to the large current competition in its feedback loops and the use of extra access transistors. However, the QCCM10T, Lin12T, and RH12T cells are less sensitive to

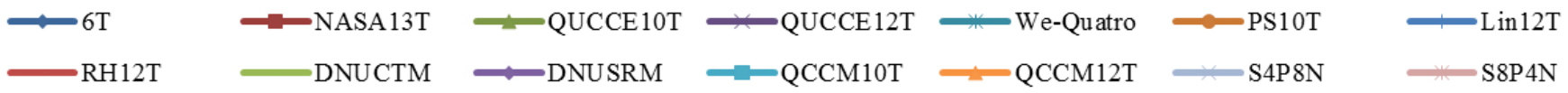

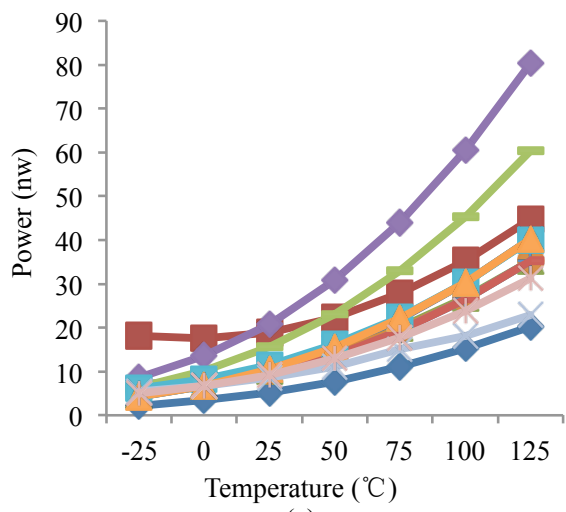

(a)

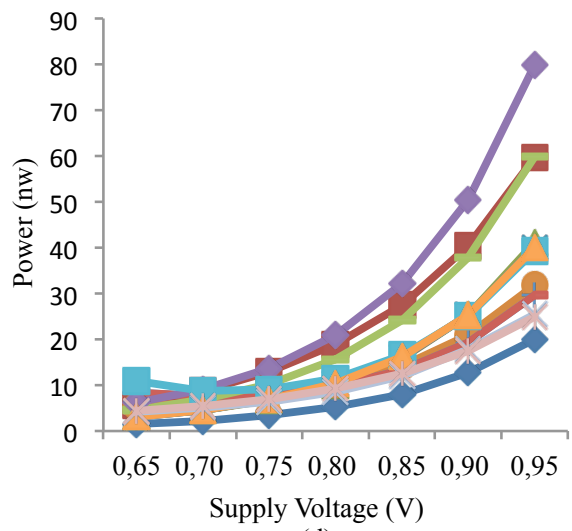

(d)

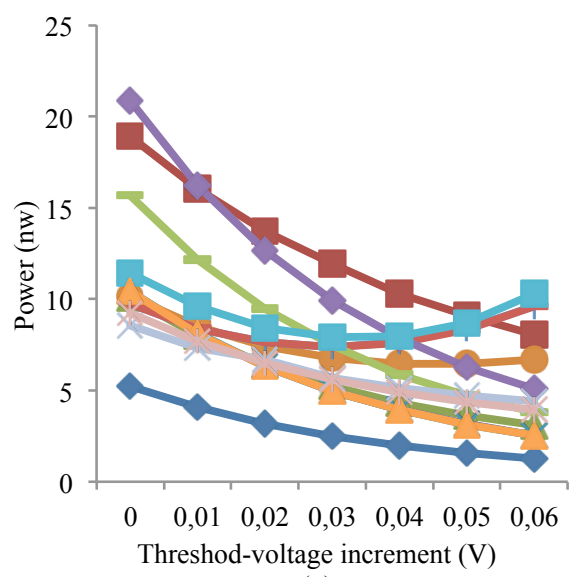

(g)

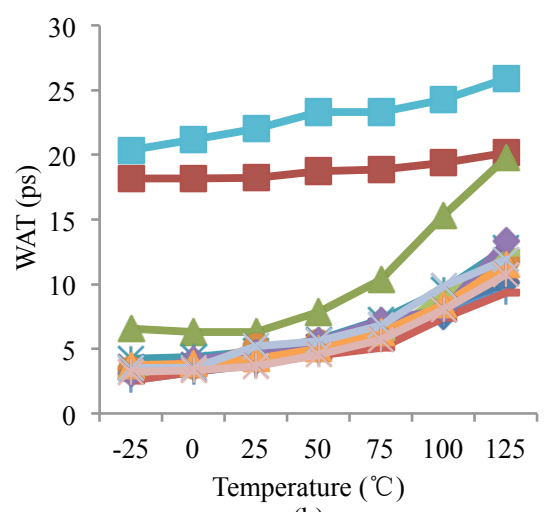

(b)

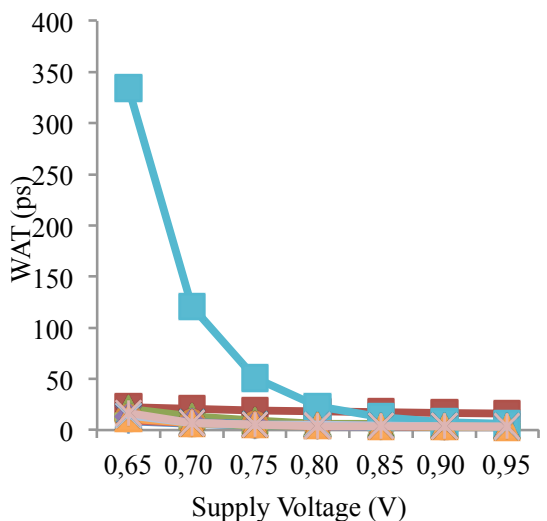

(e)

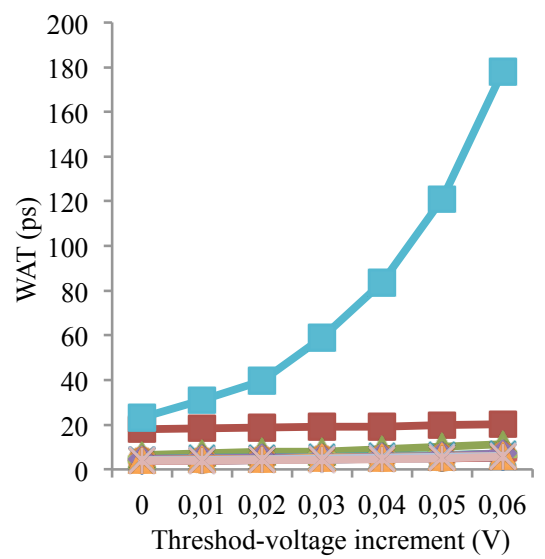

(h)

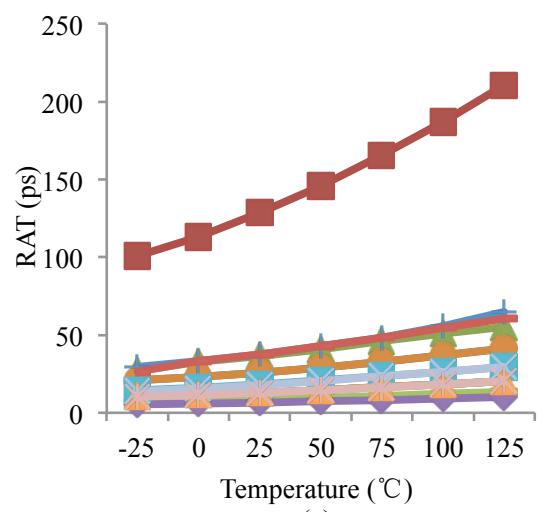

(c)

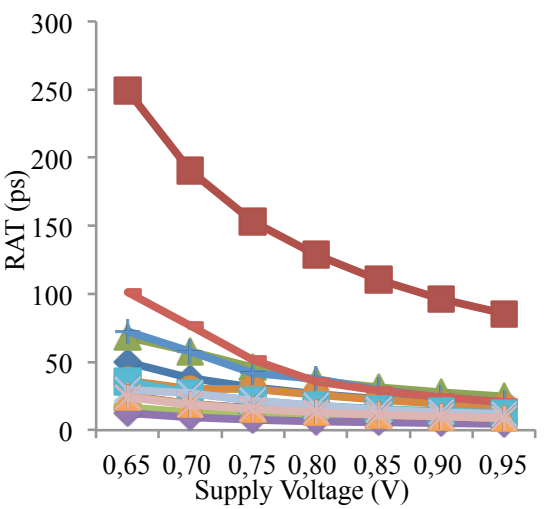

(f)

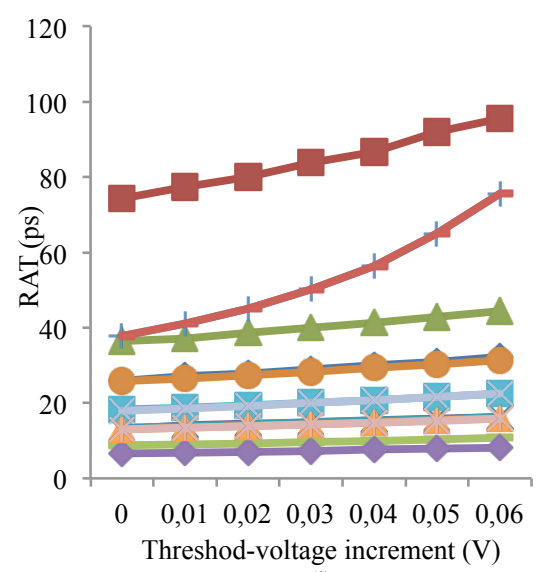

(i)

Fig. 12. Estimation results of PVT variation impacts on power, WAT, and RAT for SRAM designs. (a) Impacts of temperature variations on power. (b) Impacts of temperature variations on WAT. (c) Impacts of temperature variations on RAT. (d) Impacts of supply voltage variations on power. (e) Impacts of supply voltage variations on WAT. (f) Impacts of supply voltage variations on RAT. (g) Impacts of threshold-voltage variations on power. (h) Impacts of threshold-voltage variations on WAT. (i) Impacts of threshold-voltage variations on RAT. 
supply voltage variations on power. It can be seen from Fig 12-(h) that the QCCM10T is more sensitive to threshold voltage variation on WAT, but the variation has a low impact on the WAT of the cells, such as S4P8N and S8P4N. It can be seen from Fig 12-(i) that the RH12T and Lin12T are more sensitive to threshold voltage variation on RAT. This is mainly because they employ many devices from their storage nodes to their output. Finally, the threshold voltage variation has a low impact on the WAT of the cells, such as DNUCTM, QCCM12T, S4P8N, and S8P4N. To summarize, PVT variation has moderate impacts on power, WAT, and RAT of the proposed S4P8N and S8P4N cells, compared with the state-of-the-art hardened SRAM cells.

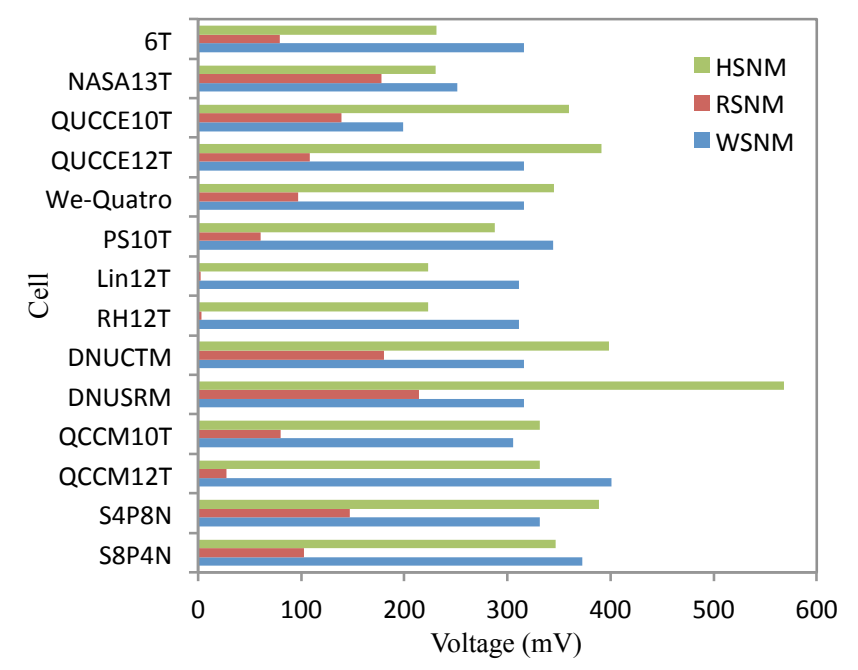

Fig. 13. SNM comparison under the supply voltage of $0.8 \mathrm{~V}$.

It is reported in [20] that static noise margin $(S N M)$ is an important metric to analyze stability of SRAM cells for normal operations. Figure 13 shows the comparison results of SNMs for different SRAM cells under a supply voltage of $0.8 \mathrm{~V}$. Note that the normal temperature was set to $25^{\circ} \mathrm{C}$. From Fig. 13, it can be seen that the hold SNM (HSNM) value of the proposed S4P8N and S8P4N cells are higher than those of the hardened NASA13T, We-Quatro, PS10T, Lin12T, RH12T, QCCM10T, and QCCM12T cells, but lower than those of the QUCCE12T, DNUCTM, DNUSRM cells. It can be seen that the read SNM (RSNM) value of the proposed S4P8N and
S8P4N cells are higher than those of the hardened We-Quatro, PS10T, Lin12T, RH12T, QCCM10T, and QCCM12T cells, but lower than those of the NASA13T, DNUCTM, DNUSRM cells. It can also be seen that the write SNM (WSNM) value of the proposed S4P8N and S8P4N cells are higher than those of the hardened NASA13T, QUCCE10T, QUCCE12T, We-Quatro, Lin12T, RH12T, DNUCTM, DNUSRM, and QCCM10T cells, but lower than those of the QCCM10T cell. To summarize, the comparison results of the SNMs show that the proposed S4P8N and S8P4N cells have moderate SNMs, compared with the state-of-the-art hardened SRAM cells.

Figure 14 shows the comparison results of SNMs under different supply voltages. Note that the normal supply voltage was set to $0.8 \mathrm{~V}$ and the supply voltage variation was ranged from $0.60 \mathrm{~V}$ to $1.30 \mathrm{~V}$. The normal temperature was still set to $25^{\circ} \mathrm{C}$. Fig. 14-(a), (b), and (c) show the impact of supply voltage variations on HSNM, RSNM, and WSNM. It can be seen that, as the supply voltage increases, the value of HSNM is generally increasing, the value of RSNM is generally decreasing, and the value of WSNM is generally increasing. It can be seen from Fig. 13-(a) that, as the supply voltage increases, the proposed S4P8N cell has a similar HSNM sensitivity compared to the DNUSRM cell, but the proposed S8P4N has a lower HSNM sensitivity. It can be seen from Fig. 14-(b) that, as the supply voltage increases, the proposed S8P4N cell has a similar RSNM sensitivity compared to the DNUCTM cell, but the proposed S4P8N has a lower RSNM sensitivity. Finally, it can be seen from Fig. 14-(c) that, as the supply voltage increases, the proposed S8P4N cell has a similar WSNM sensitivity compared to the PS10T, Lin12T, RH12T, and QCCM10T cells, but the proposed S4P8N has a lower WSNM sensitivity.

\section{CONCLUSIONS}

The continuous advancement of CMOS technologies makes SRAMs more and more sensitive to soft errors such as SNUs and DNUs. Based on the RHBD approach, two novel reliable SRAM cells, namely S4P8N and S8P4N, with optimized speed and power, have been proposed in this paper. The proposed S4P8N and S8P4N cells can self-recover from all possible SNUs and a part of DNUs. The access operations of the proposed cells are optimized through the use of four

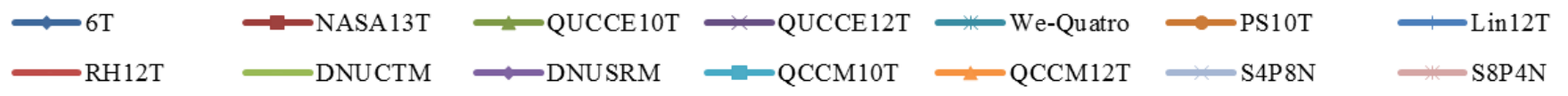

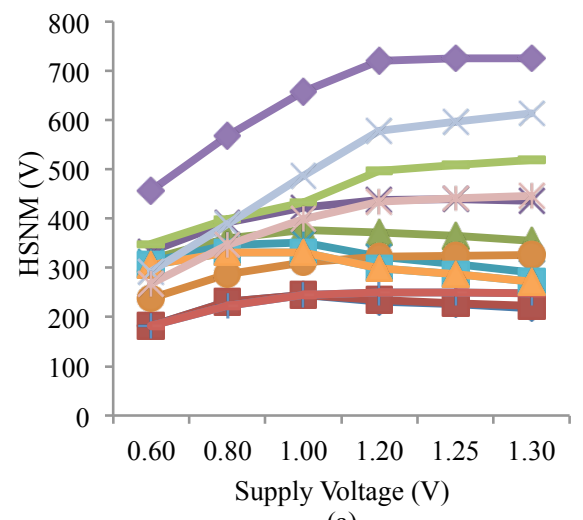

(a)

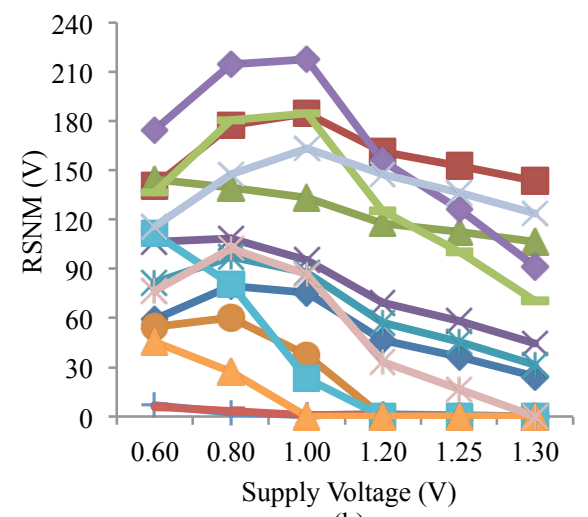

(b)

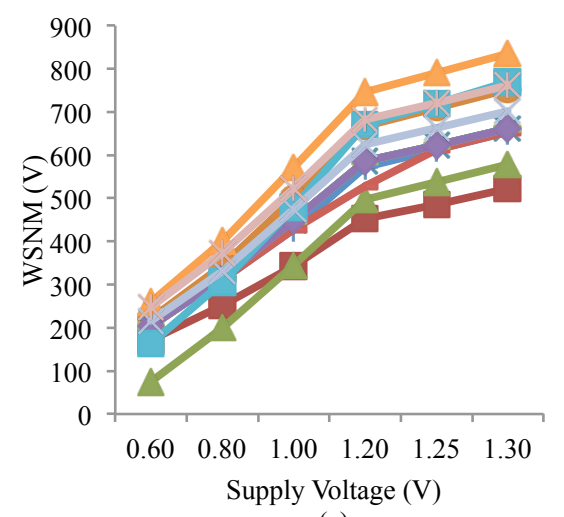

(c)

Fig. 14. SNM comparisons under different supply voltages. (a) HSNM. (b) RSNM. (c) WSNM. 
parallel access transistors. The proposed S4P8N and S8P4N cells have low overhead especially in terms of power dissipation. Compared with S4P8N, the S8P4N cell has a smaller WAT and RAT. The proposed S4P8N and S8P4N cells can be effectively applied to fields such as low-orbit aerospace and terrestrial safety-critical applications, where the DNU issue is not severe. For applications in harsh radiation environments, our previous work in [19] is suitable to provide very high reliability.

\section{REFERENCES}

[1] A. Watkins and S. Tragoudas, "Radiation hardened latch designs for double and triple node upsets," IEEE Trans. Emerg. Topics Comput., Early Access, doi: 10.1109/TETC.2017.2776285.

[2] M. Ebara, K. Yamada, K. Kojima, J. Furuta, and K. Kobayashi, "Process dependence of soft errors induced by $\alpha$ particles, heavy ions, and high energy neutrons on flip flops in FDSOI," IEEE J. Electron Devices Soc., vol. 7, no. 1, pp. 817-824, Mar. 2019, doi: 10.1109/JEDS.2019.2907299.

[3] B. Narasimham, S. Gupta, D. Reed, J. K. Wang, N. Hendrickson, and H. Tanfique, "Scaling Trends and Bias Dependence of the Soft Error Rate of $16 \mathrm{~nm}$ and $7 \mathrm{~nm}$ Fin-FET SRAMs," in Proc. Internat. Reliab. Physics Symp., Mar. 2018, pp. 1-4, doi: 10.1109/IRPS.2018.8353583.

[4] S. Cai, W. Wang, F. Yu, and B. He, "Single event transient propagation probabilities analysis for nanometer CMOS circuits," J. Electron. Testing, vol. 35, no. 2, pp. 163-172, Apr. 2019, doi: 10.1007/s10836-019-05791-2.

[5] J. Black, P. Dodd, and K. Warren, "Physics of multiple-node charge collection and impacts on single-event characterization and soft error rate prediction," IEEE Trans. Nucl. Sci, vol. 60, no. 3, pp. 1836-1851, May. 2013, doi: 10.1109/TNS.2013.2260357.

[6] A. Yan, Y. Hu, J. Song, and X. Wen, "Single-event double-upset self-recoverable and single-event transient pulse filterable latch design for low power applications," in Proc. IEEE Design, Automation Test Europe Conf., May. 2019, pp. 1658-1663, doi: 10.23919/DATE.2019.8714841.

[7] A. Yan, Z. Huang, M. Yi, X. Xu, Y. Ouyang, and H. Liang, "Double node-upset-resilient latch design for nanoscale CMOS technology," IEEE Trans. Very Large Scale Integr. (VLSI) Syst., vol. 25, no. 6, pp. 1978-1982, Jun. 2017, doi: 10.1109/TVLSI.2017.2655079.

[8] R. Rajaei, M. Tabandeh, and M. Fazeli, "Single event multiple upset (SEMU) tolerant latch designs in preSNUce of process and temperature variations," J. Circuit, Syst. Comput, vol. 24, no. 1, pp. 1-30, Sep. 2014, doi:10.1142/s0218126615500085.

[9] Y. Li, H. Wang, R. Liu, L. Chen, I. Nofal, S. T. Shi, A. L. He, G. Guo, S H. Baeg, S. J. Wen, R. Wong, M. Chen, Q. Wu, "A quatro-based $65 \mathrm{~nm}$ flip-flop circuit for soft-error resilience," IEEE Trans. Nucl. Sci., vol. 64, no. 6, pp. 1554-1561, May. 2017, doi: 10.1109/TNS.2017.2704062.

[10] B. Xia, J. Wu, H. Liu, K. Zhou, and Z. Miao, "Design and comparison of high-reliable radiation-hardened flip-flops under SMIC 40nm process," J. Circuits, Syst. Comput., vol. 25, no. 12, pp. 1-19, Dec. 2016, doi: $10.1142 / \mathrm{s} 0218126616501632$.

[11] K. Kobayashi, K. Kubota, M. Masuda, Y. Manzawa, J. Furuta, S. Kanda, and $\mathrm{H}$. Onodera, "A low-power and area-efficient radiation-hard redundant flip-flop, DICE ACFF, in a $65 \mathrm{~nm}$ thin-BOX FD-SOI," IEEE Trans. Nucl. Sci., vol. 61, no. 4, pp. 1881-1888, Jun.2014, doi: 10.1109/TNS.2014.2318326.

[12] A. Yan, J. Zhou, Y. Hu, J. Cui, Z. Huang, P. Girard, and X. Wen, "Novel Quadruple Cross-Coupled Memory Cell Designs With Protection Against Single Event Upsets and Double-Node Upsets," IEEE Access, vol. 7, pp. 176788-176196, Dec. 2009, doi: 10.1109/ACCESS.2019.2958109.

[13] Y. Shiyanovskii, A. Rajendran, and C. Papachristou, "A low power memory cell design for SEU protection against radiation effects," in Proc. Conf. Adaptive Hardware Syst., Aug. 2012, pp. 288-295, doi: 10.1109/AHS.2012.6268665.

[14] J. Jiang, Y. Xu, W. Zhu, J. Xiao, and S. Zou, "Quadruple cross-coupled latch-based 10T and 12T SRAM bit-cell designs for highly reliable terrestrial applications," IEEE Trans. Circuits Syst. I, Reg. Papers, vol. 66, no. 3, pp. 967-977, Oct. 2019, doi: 10.1109/TCSI.2018.2872507.
[15] L. Dang, J. Kim, and I. Chang, "We-Quatro: radiation-hardened SRAM cell with parametric process variation tolerance," IEEE Trans. Nucl. Sci., vol. 64, no. 9, pp. 2489-2496, Jul. 2017 doi: 10.1109/TNS.2017.2728180.

[16] I. Jung, Y. Kim, and F. Lombardi, "A novel sort error hardened 10T SRAM cells for low voltage operation," in Proc. IEEE Internat. Midwest Symp. Circuits Syst., Aug. 2012, pp. 714-717,doi: 10.1109/MWSCAS.2012.6292120.

[17] D. Lin, Y. Xu, X. Liu, et al., "A novel highly reliable and low-power radiation hardened SRAM bit-cell design," IEICE Electron. Express, vol. 15, no. 3, pp. 1-8, Feb. 2018, doi: 10.1587/elex.15.20171129.

[18] C. Hu, S. Yue, and S. Lu, "Design of a novel $12 \mathrm{~T}$ radiation hardened memory cell tolerant to single event upsets (SEU)," in Proc. IEEE 2nd Int. Conf. Integr. Circuits Microsyst, Jan. 2017, pp. 182-185, doi: 10.1109/ICAM.2017.8242164.

[19] A. Yan, Zhen. W, Jing. G, J. Song, and X. Wen, "Novel double-node-upset-tolerant memory cell designs through radiation-hardening-by-design and layout," IEEE Trans. Reliab., vol. 68, no.1, pp. 354-363, Nov. 2018, doi: 10.1109/TR.2018.2876243.

[20] C. Peng, J. Huang, C. Liu, Q. Zhao, S. Xiao, X. Wu, Z. Lin, J.Chen, and X. Zeng, "Radiation-hardened 14T SRAM bitcell with speed and power optimized for space application," IEEE Trans. Very Large Scale Integr. (VLSI) Syst., vol. 27, no. 2, pp. 407-415, Nov. 2019, doi: 10.1109/TVLSI.2018.2879341.

[21] C. Qi, L. Xiao, T. Wang, et al., "A highly reliable memory cell design combined with layout-level approach to tolerant single-event upsets," IEEE Trans. Device Mater. Reliab., vol. 16, no. 3, pp. 388-395, Jul. 2016, doi: 10.1109/TDMR.2016.2593590.

[22] J. Guo, L. Zhu, Y. Sun, H. Cao, H. Huang. T. Wang, C. Qi, R. Zhang, X. Cao, L. Xiao, and Z. Mao, "Design of area-efficient and highly reliable RHBD 10T memory cell for aerospace applications," IEEE Trans. Very Large Scale Integr. (VLSI) Syst. vol. 26, no. 5, pp. 991-994, Jan. 2018, doi: 10.1109/TVLSI.2017.2788439.

[23] G. Zhang, Y. Zeng, F. Liang, and K. Chen, "A novel SEU tolerant SRAM data cell design," IEICE Electron. Express, vol. 12, no. 17, pp. 1-6, Sep. 2015, doi: 10.1587/elex.12.20150504.

[24] N. Yadav, A. Shah, and S. Vishvakarma, "Stable, reliable and bit-interleaving 12T SRAM for space applications: a device circuit co-design," IEEE Trans. on Semicond. Manuf., vol. 30, no. 3, pp. 276-284, Jun. 2017, doi: 10.1109/TSM.2017.2718029.

[25] R. Rajaei, B. Asgari, M. Tabandeh, M. Fazeli, "Single event multiple upset-tolerant SRAM cell designs for nano-scale CMOS technology," Turkish J. Electrical Engineering Comput. Sci., vol. 25, no. 1, pp. 1053-1047, Oct. 2017, doi:10.3906/elk-1502-124.

[26] R. Rajaei, B. Asgari, M. Tabandeh, and M. Fazeil, "Design of robust SRAM cells against single-event multiple effects for nanoscale technologies," IEEE Trans. Device Mater. Reliab., vol. 15, no. 3, pp. 429-436, Jul. 2015, doi: 10.1109/TDMR.2015.2456832.

[27] T. Li, Y. Yang, J. Zhang, and J. Liu, "A novel SEU hardened SRAM bit-cell design," IEICE Electron. Express, vol. 14, no. 12, pp. 1-8, Jun. 2017, doi: 10.1587/elex.14.20170413.

[28] A. Yan, Z. Wu, J. Zhou, Y. Hu, Y. Chen, Z. Ying, X. Wen, and P. Girard, "Design of a Sextuple Cross-Coupled SRAM cell with Optimized Access Operations for Highly Reliable Terrestrial Applications," in Proc. IEEE Asian Test Symposium, Jan. 2019, pp. 1-6, doi: 10.1109/ATS47505.2019.00006.

[29] G. Messenger, "Collection of Charge on Junction Nodes from Ion Tracks," IEEE Trans. Nucl. Sci., vol. 29, no. 6, pp. 2024-2031, Dec. 1982, doi: https://dx.doi.org/10.1109/TNS.1982.4336490.

[30] A. Yan, C. Lai, Y. Zhang, J. Cui, Z. Huang, J. Song, J. Guo, and X. Wen, "Novel low cost, double-and-triple-node-upset-tolerant latch designs for nano-scale CMOS," IEEE Trans. Emerg. Topics Comput., Early Access, doi: 10.1109/TETC.2018.2871861.

[31] H. Li, L. Xiao, J. Li, and C. Qi, "High Robust and Cost Effective Double Node Upset Tolerant Latch Design for Nanoscale CMOS Technology," Microelectron. Reliab., vol. 93, pp. 89-97, Feb. 2019, doi: 10.1016/j.microrel.2019.01.005. 
Aibin Yan received a Ph. D degree in Computer Application Technology from Hefei University of Technology, and received a M.S. degree in Software Engineering from the University of Science and Technology of China, Hefei, in 2015 and 2009, respectively. In 2016, he joined Anhui University where he is currently a $\mathrm{PhD}$ supervisor and an Associate Professor. His research interests mainly include radiation hardening by design for nanoscale CMOS integrated circuits like latches, flip-flops and SRAM cells.

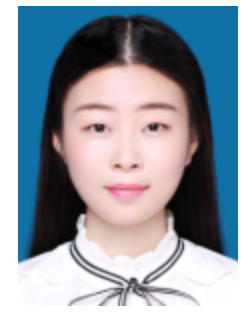

Yan Chen received her B.S degree from Anhui Institute of Information Technology in 2019. Currently, she is pursuing her M.S. degree for Computer Science and Technology major in Anhui University. Her research interests include radiation hardening by design for nano-scale CMOS ICs such as latches and SRAM cells.

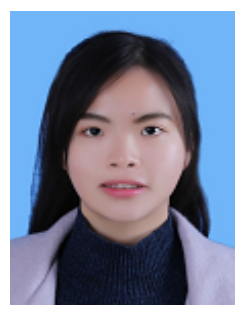

Yuanjie Hu received her B.S degree from Chuzhou Colleague in 2018. Currently, she is pursuing her M.S. degree for Software Engineering major in Anhui University. Her research interests include radiation hardening by design for nano-scale CMOS ICs such as latches and SRAM cells.

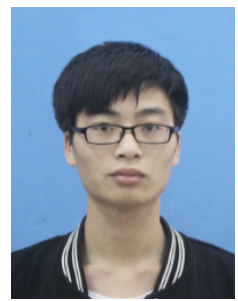

Jun Zhou received his B.S degree from Anhui University in 2017. Currently, he is pursuing his M.S. degree for Computer Technology major in Anhui University. His research interests include radiation hardening by design for nano-scale CMOS ICs such as latches and SRAM cells.

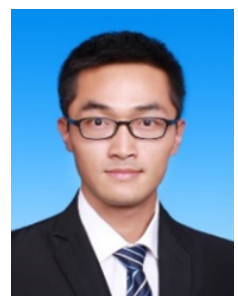

Tianming Ni received a Ph.D. degree from Hefei University of Technology, Hefei, China, in 2018. He joined the Key Laboratory of Advanced Perception and Intelligent Control of High-end Equipment, Ministry of Education, College of Electrical Engineering, Anhui Polytechnic University in 2018. His research interest includes built-in-self-test, design automation of digital systems, design for IC reliability, 3D IC test and fault tolerance.

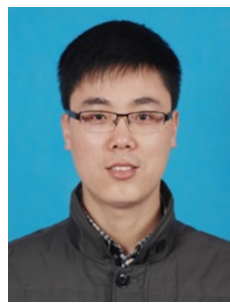

Jie Cui received a Ph.D. degree from University of Science and Technology of China, Hefei, in 2012. Currently, he is a professor with Anhui University, Hefei, Anhui, China. He has published more than 80 papers in journals and conference proceedings such as IEEE IOTJ, IEEE TIFS, IEEE TDSC, IEEE TC, IEEE TETC, IEEE TCASI, etc. His research interests include IoT security, applied cryptography, software-defined networking, vehicular ad hoc network, and design for fault tolerance.

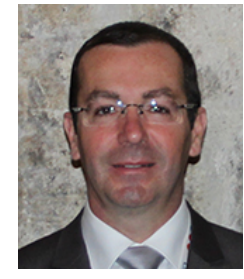

Patrick Girard received a M.Sc. degree in Electrical Engineering and a Ph.D. degree in Microelectronics from the University of Montpellier, France, in 1988 and 1992 respectively. He is currently Research Director at CNRS (French National Center for Scientific Research) and works in the Microelectronics Department of the Laboratory of Informatics, Robotics and Microelectronics of Montpellier (LIRMM) - France. From 2010 to 2014, he was head of this Microelectronics Department. He is co-Director of the International Associated Laboratory " LAFISI " (French-Italian Research Laboratory on Hardware-Software Integrated Systems) created in 2013 by the CNRS and the University of Montpellier with the Politecnico di Torino, Italy. His research interests include all aspects of digital testing and memory testing, with emphasis on critical constraints such as timing and power. Reliability and fault tolerance are also part of his research activities. He has served on numerous conference committees and is the founder and Editor-in-Chief of the ASP Journal of Low Power Electronics (JOLPE). He is also an Associate Editor of the IEEE Transactions on Computers, IEEE Transactions on Emerging Topics in Computing, and the Journal of Electronic Testing (JETTA - Springer). He has supervised $37 \mathrm{PhD}$ dissertations and has published 7 books or book chapters, 75 journal papers, and more than 250 conference and symposium papers on these fields. Patrick Girard is a Fellow of IEEE.

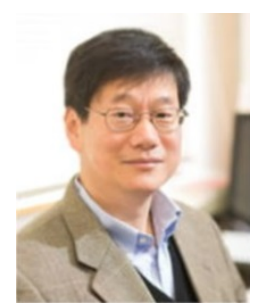

Xiaoqing Wen received the B.E. degree from Tsinghua University, China, in 1986, the M.E. degree from Hiroshima University, Japan, in 1990, and the Ph.D. degree from Osaka University, Japan, in 1993. From 1993 to 1997, he was an Assistant Professor at Akita University, Japan. He was a Visiting Researcher at University of Wisconsin, Madison, USA, from Oct. 1995 to Mar. 1996. He joined SynTest Technologies, Inc., USA, in 1998, and served as its Chief Technology Officer until 2003. In 2004, he joined Kyushu Institute of Technology, Japan, where he is currently a Professor and the Chair of the Department of Creative Informatics. He founded Dependable Integrated Systems Research Center in 2015 and served as its Director until 2017. His research interests include VLSI test, diagnosis, and testable design. He co-authored and co-edited two books: VLSI Test Principles and Architectures: Design for Testability (Morgan Kaufmann, 2006) and Power-Aware Testing and Test Strategies for Low Power Devices (Springer, 2009). He holds 43 U.S. Patents and 14 Japan Patents on VLSI testing. He received the 2008 IEICE-ISS Best Paper Award for his pioneering work on $\mathrm{X}$-filling-based low-capture-power test generation. He is a Fellow of the IEEE, a member of the IEICE, the IPSJ, and the REAJ. He has/is served/serving as associate editors for IEEE Transactions on Computer-Aided Design, IEEE Transactions on very large scale integration (VLSI) systems, and the Journal of Electronic Testing: Theory and Applications. 